This is the peer reviewed version of the following article: "Witczak, M, Buciakowski, M, Puig, V, Rotondo, D, and Nejjari, F (2015), An LMI approach to robust fault estimation for a class of nonlinear systems. Int. J. Robust. Nonlinear Control" which has been published in final form at [doi: 10.1002/rnc.3365]. This article may be used for non-commercial purposes in accordance with Wiley Terms and Conditions for Self-Archiving." 


\title{
An LMI approach to robust fault estimation for a class of non-linear systems
}

\author{
Marcin Witczak ${ }^{1 *}$ Mariusz Buciakowski ${ }^{1}$, Vicenç Puig ${ }^{2}$, Damiano Rotondo ${ }^{2}$, \\ Fatiha Nejjari $^{2}$ \\ ${ }^{1}$ Institute of Control and Computation Engineering, University of Zielona Góra, ul. Podgórna 50, 65-246 \\ Zielona Góra, Poland. \\ ${ }^{2}$ Automatic Control Department, Universidad Politécnica de Cataluña, Rambla Sant Nebridi, 10, 08222 Terrassa, \\ Spain.
}

\begin{abstract}
SUMMARY
The paper presents a robust fault estimation approach for a class of non-linear discrete-time systems. In particular, two sources of uncertainty are present in the considered class of systems, i.e., an unknown input and an exogenous external disturbance. Thus, apart from simultaneous state and fault estimation, the objective is to decouple the effect of an unknown input while minimizing the influence of the exogenous external disturbance within the $\mathcal{H}_{\infty}$ framework. The resulting design procedure guarantees that a prescribed disturbance attenuation level is achieved with respect to the state and fault estimation error while assuring the convergence of the observer. The core advantage of the proposed approach is its simplicity by reducing the fault estimation problem to matrix inequalities formulation. In addition, the design conditions ensure the convergence of the observer with guaranteed $\mathcal{H}_{\infty}$ performance. The effectiveness of the proposed approach is demonstrated by its application to a Twin Rotor MIMO System.
\end{abstract}

Received ...

KEY WORDS: Fault diagnosis, fault identification, robust estimation, non-linear systems, observers, linear matrix inequalities.

\section{INTRODUCTION}

Fault diagnosis aims at deciding if a fault has occurred (fault detection), finding in which component the fault is located (fault isolation), and identifying the fault by estimating the size (fault identification and estimation) [3]. Thus, fault diagnosis can be seen as a three-step procedure, which includes fault detection, isolation and identification. In the literature, the problem of fault detection and isolation (FDI) has been studied widely. For an overview of the main approaches the reader is referred to several books, e.g. [12, 5, 18, 9, 23, 47].

However, the fault identification and estimation task has been studied less, until recent years when it has been addressed due to its link with the active Fault-Tolerant Control (FTC) problem [3]. In case that a fault accommodation strategy is needed, an active FTC requires on-line fault diagnosis including not only FDI but also fault identification and estimation. Otherwise, without this knowledge, an appropriate compensation of the fault effect is impossible.

\footnotetext{
${ }^{*}$ Correspondence to: Marcin Witczak, Institute of Control and Computation Engineering, University of Zielona Góra, ul. Podgórna 50, 65-246 Zielona Góra, Poland, e-mail: M.Witczak@issi.uz.zgora.pl

${ }^{\dagger}$ The work was financed as a research project with the science funds for the years 2014-2017. This work has also been partially funded by the Spanish Ministry of Science and Technology through the projects CICYT ECOCIS (ref. DPI201348243-C2-1-R) and CICYT HARCRICS (ref. DPI2014-58104-R), by AGAUR through the contract FI-DGR 2014 (ref. 2014FI_B1_00172), and by the DGR of the Generalitat de Catalunya (ref. 2014/SGR/374)
} 
Many books have been published in the last decade on the emerging problem of FTC, which deal with fault identification and estimation problems. In particular, [19], which is mainly devoted to fault diagnosis and its applications, provides some general rules for hardware-redundancy-based FTC. On the other hand, the concepts of achieving passive FTC are introduced in [26], where the authors also investigate the problem of performance and stability of FTC under imperfect fault diagnosis. In particular, they consider (under some assumptions) the effect of delayed fault detection and imperfect fault identification. However, the fault diagnosis scheme is treated separately during the design and no real integration of fault diagnosis and FTC is proposed. FTC is also treated in [30], where a number of practical FTC case studies is presented, i.e., a winding machine, a threetank system, and an active suspension system.

The problem of fault estimation, which can also be perceived as the estimation of an unknown input, has been addressed using different strategies. Some of these strategies deserve particular attention, namely: augmenting the state vector by an unknown input, two-stage Kalman filter [21], minimum variance input and state estimator [13, 22], adaptive estimation [52], sliding mode highgain observers [46] and finally, an $\mathcal{H}_{\infty}$ approach [29]. The fault estimation can also be formulated as a parameter estimation problem [39] leading to the application of any parameter estimation algorithms such as least squares, generalized/extended least squares or instrumental variables. Recently, a lot of effort has been devoted to extend the previous approaches to non-linear systems. For example, [6] proposed a unified framework based on a model reference approach for non-linear systems that can be represented by means of Takagi-Sugeno models. In [41], a fault estimation scheme for non-linear systems that can be modeled in linear parameter varying (LPV) form is presented. In [45], an observer scheme that estimates simultaneously the state and the fault is presented. On the other hand, the robustness of the fault estimation algorithm can be stated as the degree to which the sensitivity of the fault estimates is invariant in the presence of model parameter variations, disturbances, uncertainty and noise.

For non-linear systems, the observer-based FDI approaches have gained a lot of interest [35, 2] and FDI formulations for some classes of non-linear systems have been derived. In [16], state-affine non-linear systems have been handled, and in [34, 14], the class of input-affine systems has been considered, among others. The work [33] presents a detailed geometric description of how to tackle the residual generation problem for non-linear systems. On the other hand, [25] presents a procedure to design a bank of extended $\mathcal{H}_{\infty}$ observers for sensor FDI for a certain class of non-linear systems. There are also approaches that employ soft computing techniques, e.g. neural networks [28].

In this paper, a robust fault estimation approach for discrete-time non-linear systems based on the general idea of Unknown Input Observer (UIO) [11, 47] and the $\mathcal{H}_{\infty}$ framework is proposed, in order to address the issue of robustness in the presence of uncertainty and disturbances.

A large amount of knowledge on using these techniques for model-based fault diagnosis has been acquired for the last three decades and can be accessed through the literature (see, e.g., [47] and the references therein). As an example, the high gain observer for Lipschitz systems was applied for the purpose of fault diagnosis in [17]. One of the standard methods for observer design, which can be applied for fault diagnosis and FTC, consists in using the non-linear change of coordinates to bring the original system into a linear one (a pseudo linear one) [16, 20]. It is worth noting that there are efficient approaches for bilinear systems [15]. It should be also pointed out that, when the feasibility condition of the above schemes are not matched, then the well known Extended Kalman Filter (EKF) can be applied in both a stochastic and a deterministic context (see, e.g., [47]). However, its main drawback is related to problems with its convergence. Concerning fault estimation schemes, some interesting approaches were proposed for a multi-model description of non-linear systems. In particular, in [40], the authors introduced a fault estimator that is based on the output error and its derivative. The resulting derivative term of the fault is treated as a bounded disturbance and its effect is suitably minimized, and finally the scheme is formed within the convenient augmented structure (cf. [32, 31]). An interesting structure was proposed in [43]. Along with fault estimation, it guarantees robust performance with respect to the output error. Apart from augmented structures, there are methods that employ adaptive or sliding mode paradigms (see, e.g. [44, 46]). 
The approach proposed in this work can be perceived as a combination of linear-system strategies [13, 29] for a class of non-linear systems [42, 51]. It should also be pointed out that the proposed description of non-linearity constitutes an alternative to other approaches presented in the literature [1, 27, 37, 36, 33].

The main contribution of this paper is the novel robust state and fault estimator design procedure. In particular, two sources of uncertainty are present in the system being considered, i.e., an unknown input and an exogenous external disturbance. Thus, apart from simultaneous state and fault estimation, the objective is to decouple the effect of an unknown input as well as to minimize the influence of the exogenous external disturbance within the $\mathcal{H}_{\infty}$ framework. The resulting design procedure guarantees that a prescribed disturbance attenuation level is achieved with respect to the state and fault estimation error while assuring the convergence of the observer. The core advantage of the proposed approach is its simplicity by reducing the fault estimation problem to matrix inequalities formulation. In addition, the design conditions ensure the convergence of the observer with guaranteed $\mathcal{H}_{\infty}$ performance. Furthermore, it is shown that a solution can be found by solving a set of Linear Matrix Inequalities (LMIs), a problem for which efficient solvers are available nowadays. The effectiveness of the proposed method is demonstrated through its application to a two degree of freedom helicopter subject to actuator faults. It will be shown that these results clearly recommend the proposed approach for the purpose of active FTC [7, 10, 49].

The paper is organised as follows. Section 2 presents preliminaries regarding the problem being undertaken. In Section 3, the robust fault estimation approach is proposed while in Section 4, the unknown input design procedure is described. Section 5 presents the application of the proposed approach to a Twin Rotor MIMO System (TRMS), showing its effectiveness and performance. Finally, the last section concludes the paper.

\section{PRELIMINARIES}

Let us consider a non-linear discrete-time system

$$
\begin{aligned}
\boldsymbol{x}_{k+1} & =\boldsymbol{A} \boldsymbol{x}_{k}+\boldsymbol{B} \boldsymbol{u}_{k}+\boldsymbol{D} \boldsymbol{d}_{k}+\boldsymbol{g}\left(\boldsymbol{x}_{k}, \boldsymbol{u}_{k}\right)+\boldsymbol{B} \boldsymbol{f}_{k}+\boldsymbol{W}_{1} \boldsymbol{w}_{k}, \\
\boldsymbol{y}_{k} & =\boldsymbol{C} \boldsymbol{x}_{k}+\boldsymbol{W}_{2} \boldsymbol{w}_{k},
\end{aligned}
$$

where $\boldsymbol{x}_{k} \in \mathbb{X} \subset \mathbb{R}^{n}$ is the state, $\boldsymbol{u}_{k} \in \mathbb{U} \in \mathbb{R}^{r}$ stands for the input, $\boldsymbol{y}_{k} \in \mathbb{R}^{m}$ denotes the output, $\boldsymbol{f}_{k} \in \mathbb{R}^{r}$ stands for the fault, $\boldsymbol{d}_{k} \in \mathbb{R}^{q}$ is the unknown input disturbance, $\boldsymbol{w}_{k} \in l_{2}$ is a an exogenous disturbance vector satisfying

$$
l_{2}=\left\{\mathbf{w} \in \mathbb{R}^{n} \mid\|\mathbf{w}\|_{l_{2}}<+\infty\right\},\|\mathbf{w}\|_{l_{2}}=\left(\sum_{k=0}^{\infty}\left\|\boldsymbol{w}_{k}\right\|^{2}\right)^{\frac{1}{2}} .
$$

Finally, $\boldsymbol{W}_{1} \in \mathbb{R}^{n \times n}$ and $\boldsymbol{W}_{2} \in \mathbb{R}^{m \times n}$ stand for the exogenous disturbance distribution matrices.

For the purpose of this work, the following assumptions are considered:

Assumption 1: There exists a matrix $M$ such that

$$
(\boldsymbol{g}(\boldsymbol{a}, \boldsymbol{u})-\boldsymbol{g}(\boldsymbol{b}, \boldsymbol{u}))^{T}(\boldsymbol{a}-\boldsymbol{b}) \leq(\boldsymbol{a}-\boldsymbol{b})^{T} \boldsymbol{M}(\boldsymbol{a}-\boldsymbol{b}), \quad \forall \boldsymbol{a}, \boldsymbol{b} \in \mathbb{X}, \boldsymbol{u} \in \mathbb{U} .
$$

Assumption 2: There exists a matrix $M$ such that

$$
(\boldsymbol{g}(\boldsymbol{a}, \boldsymbol{u})-\boldsymbol{g}(\boldsymbol{b}, \boldsymbol{u}))^{T}(\boldsymbol{g}(\boldsymbol{a}, \boldsymbol{u})-\boldsymbol{g}(\boldsymbol{b}, \boldsymbol{u})) \leq(\boldsymbol{a}-\boldsymbol{b})^{T} \boldsymbol{M}^{T} \boldsymbol{M}(\boldsymbol{a}-\boldsymbol{b}), \quad \forall \boldsymbol{a}, \boldsymbol{b} \in \mathbb{X}, \boldsymbol{u} \in \mathbb{U} .
$$

Assumption 3: The fault satisfies

$$
\varepsilon_{k}=\boldsymbol{f}_{k+1}-\boldsymbol{f}_{k}, \quad \varepsilon_{k} \in l_{2} .
$$


Assumption 4: The following rank condition is satisfied

$$
\operatorname{rank}(\boldsymbol{D})=\operatorname{rank}(\boldsymbol{C D})=q, \quad q \leq m .
$$

It is worth noting that, if $\boldsymbol{M}^{T} \boldsymbol{M}=\gamma^{2} \boldsymbol{I}$ then the relation underlying Assumption 2 (cf. [42, 51]) becomes a usual Lipschitz condition $[1,27,37,36]$ with $\gamma$ being a Lipschitz constant. This appealing property makes the employed strategy more general than those presented in the literature $[1,27,37$, 36]. Moreover, a significant progress was recently obtained in the observer design for non-linear systems by introducing the so-called one-sided Lipschitz condition [53], which means that a wider spectrum of systems can be tackled with the new approach. Indeed, if $\boldsymbol{M}=\zeta \boldsymbol{I}$, then the relation underlying Assumption 1 becomes a usual one-sided Lipschitz condition, which is imposed along with the usual Lipschitz condition (see [53] for further details and explanations). Thus, it is evident that this appealing property makes again the employed strategy more general than those presented in the literature (see, [53] and the references therein). Finally, Assumption 3 is required to attain a suitable fault estimation quality while Assumption 4 is used to decouple the effect of an unknown input (see, e.g., [13, 4] for further details).

Subsequently, using the Differential Mean Value Theorem (DMVT) [50], it can be shown that

$$
\boldsymbol{g}(\boldsymbol{a}, \boldsymbol{u})-\boldsymbol{g}(\boldsymbol{b}, \boldsymbol{u})=\boldsymbol{M}_{x, u}(\boldsymbol{a}-\boldsymbol{b}),
$$

with

$$
\boldsymbol{M}_{x, u}=\left[\begin{array}{c}
\frac{\partial g_{1}}{\partial x}\left(\boldsymbol{c}_{\boldsymbol{1}}, \boldsymbol{u}\right) \\
\vdots \\
\frac{\partial g_{n}}{\partial x}\left(\boldsymbol{c}_{\boldsymbol{n}}, \boldsymbol{u}\right)
\end{array}\right],
$$

where $\boldsymbol{c}_{1}, \ldots, \boldsymbol{c}_{n} \in \operatorname{Co}(\boldsymbol{a}, \boldsymbol{b}), \boldsymbol{c}_{i} \neq \boldsymbol{a}, \boldsymbol{c}_{i} \neq \boldsymbol{b}, i=1, \ldots, n$. Assuming that

$$
\bar{a}_{i, j} \geq \frac{\partial g_{i}(\boldsymbol{x})}{\partial x_{j}} \geq \underline{a}_{i, j}, \quad i=1, \ldots, n, \quad j=1, \ldots, n,
$$

it is clear that there exists a matrix $M \in \mathbb{M}$ such that

$$
\mathbb{M}=\left\{\boldsymbol{M} \in \mathbb{R}^{n \times n} \mid \bar{a}_{i, j} \geq m_{i, j} \geq \underline{a}_{i, j}, i, j=1, \ldots, n,\right\},
$$

which means that conditions of Assumptions 1 and Assumptions 2 can be satisfied.

Given the above preliminaries and assumptions, the objective of the subsequent part of this paper is to provide a novel state and fault estimation strategy for the class of non-linear discrete-time systems (1)-(2). The main advantage of the proposed approach is the fact that apart from estimating simultaneously the fault and the state, it is able to decouple the effect of unknown inputs and minimize the influence of external disturbances within the $\mathcal{H}_{\infty}$ framework.

\section{FAULT ESTIMATION STRATEGY}

Considering the system model (1)-(2), the problem is to design an observer that will be able to estimate simultaneously the state $\boldsymbol{x}_{k}$ and the fault $\boldsymbol{f}_{k}$, and decouple the effect of the unknown input $\boldsymbol{d}_{k}$. For that purpose, the following structure is proposed

$$
\begin{aligned}
\boldsymbol{z}_{k+1} & =\boldsymbol{N} \boldsymbol{z}_{k}+\boldsymbol{G} \boldsymbol{u}_{k}+\boldsymbol{L} \boldsymbol{y}_{k}+\boldsymbol{T} \boldsymbol{B} \hat{\boldsymbol{f}}_{k}+\boldsymbol{T} \boldsymbol{g}\left(\hat{\boldsymbol{x}}_{k}, \boldsymbol{u}_{k}\right), \\
\hat{\boldsymbol{x}}_{k} & =\boldsymbol{z}_{k}-\boldsymbol{E} \boldsymbol{y}_{k}, \\
\hat{\boldsymbol{f}}_{k+1} & =\hat{\boldsymbol{f}}_{k}+\boldsymbol{F}\left(\boldsymbol{y}_{k}-\boldsymbol{C} \hat{\boldsymbol{x}}_{k}\right) .
\end{aligned}
$$

Using (12)-(14), the state estimation error can be formulated as follows

$$
\boldsymbol{e}_{k}=\boldsymbol{x}_{k}-\hat{\boldsymbol{x}}_{k}=\boldsymbol{x}_{k}-\boldsymbol{z}_{k}+\boldsymbol{E} \boldsymbol{C} \boldsymbol{x}_{k}+\boldsymbol{E} \boldsymbol{W}_{2} \boldsymbol{w}_{k}=\boldsymbol{T} \boldsymbol{x}_{k}-\boldsymbol{z}_{k}+\boldsymbol{E} \boldsymbol{W}_{2} \boldsymbol{w}_{k},
$$


where

$$
T=I+E C
$$

and hence

$$
\boldsymbol{z}_{k}=\boldsymbol{T} \boldsymbol{x}_{k}+\boldsymbol{E} \boldsymbol{W}_{2} \boldsymbol{w}_{k}-\boldsymbol{e}_{k} .
$$

Following (15), the state estimation error obeys

$$
\boldsymbol{e}_{k+1}=\boldsymbol{T} \boldsymbol{x}_{k+1}-\boldsymbol{z}_{k+1}+\boldsymbol{E} \boldsymbol{W}_{2} \boldsymbol{w}_{k+1} .
$$

Substituting (1) and (12) into (18) gives the state estimation error dynamics

$$
\begin{aligned}
\boldsymbol{e}_{k+1} & =\boldsymbol{N} \boldsymbol{e}_{k}+[\boldsymbol{T} \boldsymbol{A}-\boldsymbol{N} \boldsymbol{T}-\boldsymbol{L} \boldsymbol{C}] \boldsymbol{x}_{k}+\left[\boldsymbol{T} \boldsymbol{W}_{1}-\boldsymbol{N} \boldsymbol{E} \boldsymbol{W}_{2}-\boldsymbol{L} \boldsymbol{W}_{2}\right] \boldsymbol{w}_{k} \\
& +\boldsymbol{T} \boldsymbol{B}\left[\boldsymbol{f}_{k}-\hat{\boldsymbol{f}}_{k}\right]+\boldsymbol{T}\left[\boldsymbol{g}\left(\boldsymbol{x}_{k}, \boldsymbol{u}_{k}\right)-\boldsymbol{g}\left(\hat{\boldsymbol{x}}_{k}, \boldsymbol{u}_{k}\right)\right] \\
& +[\boldsymbol{T} \boldsymbol{B}-\boldsymbol{G}] \boldsymbol{u}_{k}+\boldsymbol{T} \boldsymbol{D} \boldsymbol{d}_{k}+\boldsymbol{E} \boldsymbol{W}_{2} \boldsymbol{w}_{k+1} .
\end{aligned}
$$

From (19), it is evident that to attain the unknown input decoupling, it is necessary to set

$$
T D=0,
$$

which, considering (16), is equivalent to

$$
[\boldsymbol{I}+\boldsymbol{E} C] \boldsymbol{D}=\mathbf{0},
$$

and under Assumption 4 leads to:

$$
\boldsymbol{E}=-\boldsymbol{D}\left[(\boldsymbol{C D})^{T}(\boldsymbol{C D})\right]^{-1}(\boldsymbol{C D})^{T} .
$$

Similarly, setting

$$
\begin{gathered}
\boldsymbol{T} \boldsymbol{A}=\boldsymbol{N} \boldsymbol{T}+\boldsymbol{L} \boldsymbol{C}, \\
\boldsymbol{T} \boldsymbol{B}-\boldsymbol{G}=\mathbf{0}, \\
\boldsymbol{s}_{k}=\boldsymbol{g}\left(\boldsymbol{x}_{k}, \boldsymbol{u}_{k}\right)-\boldsymbol{g}\left(\hat{\boldsymbol{x}}_{k}, \boldsymbol{u}_{k}\right),
\end{gathered}
$$

with the fault estimation error defined as

$$
e_{f, k}=\boldsymbol{f}_{k}-\hat{\boldsymbol{f}}_{k}
$$

gives

$$
\boldsymbol{e}_{k+1}=\boldsymbol{N} \boldsymbol{e}_{k}+\boldsymbol{T} \boldsymbol{s}_{k}+\boldsymbol{T} \boldsymbol{B} \boldsymbol{e}_{f, k}+\left[\boldsymbol{T} \boldsymbol{W}_{1}-\boldsymbol{N} \boldsymbol{E} \boldsymbol{W}_{2}-\boldsymbol{L} \boldsymbol{W}_{2}\right] \boldsymbol{w}_{k}+\boldsymbol{E} \boldsymbol{W}_{2} \boldsymbol{w}_{k+1} .
$$

Substituting $\boldsymbol{T}=\boldsymbol{I}+\boldsymbol{E} \boldsymbol{C}$ into (23) yields

$$
N=T A-N E C-L C=T A-K C,
$$

where

$$
\boldsymbol{K}=\boldsymbol{N E}+\boldsymbol{L} .
$$

From (29), it follows that:

$$
\boldsymbol{e}_{k+1}=\boldsymbol{N} \boldsymbol{e}_{k}+\boldsymbol{T} \boldsymbol{s}_{k}+\boldsymbol{T} \boldsymbol{B} \boldsymbol{e}_{f, k}+\left[\boldsymbol{T} \boldsymbol{W}_{1}-[\boldsymbol{N} \boldsymbol{E}+\boldsymbol{L}] \boldsymbol{W}_{2}\right] \boldsymbol{w}_{k}+\boldsymbol{E} \boldsymbol{W}_{2} \boldsymbol{w}_{k+1} .
$$

Bearing in mind that $\boldsymbol{N E}+\boldsymbol{L}=\boldsymbol{N} \boldsymbol{E}+\boldsymbol{K}-\boldsymbol{N} \boldsymbol{E}=\boldsymbol{K}$, equation (30) can be written as follows

$$
\boldsymbol{e}_{k+1}=[\boldsymbol{T} \boldsymbol{A}-\boldsymbol{K} \boldsymbol{C}] \boldsymbol{e}_{k}+\boldsymbol{T} \boldsymbol{s}_{k}+\boldsymbol{T} \boldsymbol{B} \boldsymbol{e}_{f, k}+\left[\boldsymbol{T} \boldsymbol{W}_{1}-\boldsymbol{K} \boldsymbol{W}_{2}\right] \boldsymbol{w}_{k}+\boldsymbol{E} \boldsymbol{W}_{2} \boldsymbol{w}_{k+1} .
$$


Considering the state estimation error description, a fault estimation error can be described in a similar manner

$$
\begin{aligned}
\boldsymbol{e}_{f, k+1} & =\boldsymbol{f}_{k+1}-\hat{\boldsymbol{f}}_{k+1} \\
& =\boldsymbol{f}_{k+1}-\hat{\boldsymbol{f}}_{k}-\boldsymbol{F} \boldsymbol{C} \boldsymbol{e}_{k}-\boldsymbol{F} \boldsymbol{W}_{2} \boldsymbol{w}_{k} \\
& =\boldsymbol{f}_{k+1}-\boldsymbol{f}_{k}+\boldsymbol{f}_{k}-\hat{\boldsymbol{f}}_{k}-\boldsymbol{F} \boldsymbol{C} \boldsymbol{e}_{k}-\boldsymbol{F} \boldsymbol{W}_{2} \boldsymbol{w}_{k} \\
& =-\boldsymbol{F} \boldsymbol{C} \boldsymbol{e}_{k}+\boldsymbol{e}_{f, k}+\boldsymbol{\varepsilon}_{k}-\boldsymbol{F} \boldsymbol{W}_{2} \boldsymbol{w}_{k} .
\end{aligned}
$$

The state (31) and fault (32) estimation errors can be described in a condensed form by defining some new variables

$$
\begin{aligned}
\overline{\boldsymbol{e}}_{k+1} & =\left[\boldsymbol{e}_{k+1}^{T}, \boldsymbol{e}_{f, k+1}^{T}\right]^{T}, \\
\boldsymbol{v}_{k} & =\left[\boldsymbol{w}_{k}^{T}, \boldsymbol{\varepsilon}_{k}^{T}, \boldsymbol{w}_{k+1}^{T}\right]^{T},
\end{aligned}
$$

which leads to the following relation

$$
\overline{\boldsymbol{e}}_{k+1}=\left[\begin{array}{cc}
\boldsymbol{T} \boldsymbol{A}-\boldsymbol{K C} & \boldsymbol{T} \boldsymbol{B} \\
-\boldsymbol{F} \boldsymbol{C} & \boldsymbol{I}
\end{array}\right] \overline{\boldsymbol{e}}_{k}+\left[\begin{array}{c}
\boldsymbol{T} \\
\mathbf{0}
\end{array}\right] \boldsymbol{s}_{k}+\left[\begin{array}{ccc}
\boldsymbol{T} \boldsymbol{W}_{1}-\boldsymbol{K} \boldsymbol{W}_{2} & \mathbf{0} & \boldsymbol{E} \boldsymbol{W}_{2} \\
-\boldsymbol{F} \boldsymbol{W}_{2} & \boldsymbol{I} & \mathbf{0}
\end{array}\right] \boldsymbol{v}_{k}
$$

For the purpose of further analysis, equation (35) can be described in a more compact form

$$
\overline{\boldsymbol{e}}_{k+1}=\boldsymbol{X} \overline{\boldsymbol{e}}_{k}+\boldsymbol{Y} \boldsymbol{s}_{k}+\boldsymbol{Z} \boldsymbol{v}_{k},
$$

where

$$
\begin{aligned}
\boldsymbol{X} & =\overline{\boldsymbol{A}}-\overline{\boldsymbol{K}} \overline{\boldsymbol{C}}=\left[\begin{array}{cc}
\boldsymbol{T} \boldsymbol{A} & \boldsymbol{T} \boldsymbol{B} \\
\mathbf{0} & \boldsymbol{I}
\end{array}\right]-\left[\begin{array}{c}
\boldsymbol{K} \\
\boldsymbol{F}
\end{array}\right]\left[\begin{array}{ll}
\boldsymbol{C} & \mathbf{0}
\end{array}\right], \\
\boldsymbol{Y} & =\left[\begin{array}{c}
\boldsymbol{T} \\
\mathbf{0}
\end{array}\right], \\
\boldsymbol{Z} & =\overline{\boldsymbol{W}}-\overline{\boldsymbol{K}} \overline{\boldsymbol{V}}=\left[\begin{array}{ccc}
\boldsymbol{T} \boldsymbol{W}_{1} & \mathbf{0} & \boldsymbol{E} \boldsymbol{W}_{2} \\
\mathbf{0} & \boldsymbol{I} & \mathbf{0}
\end{array}\right]-\left[\begin{array}{c}
\boldsymbol{K} \\
\boldsymbol{F}
\end{array}\right]\left[\begin{array}{lll}
\boldsymbol{W}_{2} & \mathbf{0} & \mathbf{0}
\end{array}\right] .
\end{aligned}
$$

Taking into account the evolution of both state and fault estimation errors (36), it is possible to provide a new observer design procedure, which is comprehensively detailed in the subsequent section.

\section{OBSERVER DESIGN}

The following theorem provides the procedure for designing the observer for the system (1)-(2) using the approach proposed in the previous section:

Theorem 1

For a prescribed $\boldsymbol{v}_{k}$ attenuation level $\mu$, the observer design problem for the system (1)-(2) is solvable if there exist $\boldsymbol{N}, \boldsymbol{U}, \boldsymbol{P} \succ \mathbf{0}, \alpha>0, \beta>0$ such that for all $\boldsymbol{M} \in \mathbb{M}$ the following condition is satisfied:

$$
\left[\begin{array}{ccccc}
\boldsymbol{I}-\boldsymbol{P}+\alpha \boldsymbol{V}^{T}\left(\boldsymbol{M}+\boldsymbol{M}^{T}\right) \boldsymbol{V}^{T} & -\alpha \boldsymbol{V}^{T} & \mathbf{0} & \overline{\boldsymbol{A}}^{T} \boldsymbol{P}-\overline{\boldsymbol{C}}^{T} \boldsymbol{N}^{T} & \boldsymbol{V}^{T} \boldsymbol{M}^{T} \boldsymbol{U}^{T} \\
-\alpha \boldsymbol{V} & -\beta \boldsymbol{I} & \mathbf{0} & \boldsymbol{Y}^{T} \boldsymbol{P} & \mathbf{0} \\
\mathbf{0} & \mathbf{0} & -\mu^{2} \boldsymbol{I} & \overline{\boldsymbol{W}}^{T} \boldsymbol{P}-\overline{\boldsymbol{V}}^{T} \boldsymbol{N}^{T} & \mathbf{0} \\
\boldsymbol{P} \overline{\boldsymbol{A}}-\boldsymbol{N} \overline{\boldsymbol{C}} & \boldsymbol{P} \boldsymbol{Y} & \boldsymbol{P} \overline{\boldsymbol{W}}-\boldsymbol{N} \overline{\boldsymbol{V}} & -\boldsymbol{P} & \mathbf{0} \\
\boldsymbol{U} \boldsymbol{M} \boldsymbol{V} & \mathbf{0} & \mathbf{0} & \mathbf{0} & \beta \boldsymbol{I}-\boldsymbol{U}-\boldsymbol{U}^{T}
\end{array}\right] \prec \mathbf{0},
$$

with $\boldsymbol{N}=\boldsymbol{P} \overline{\boldsymbol{K}}$. 
Proof

The problem of $\mathcal{H}_{\infty}$ observer design [24] is to determine the matrices $\boldsymbol{N}, \boldsymbol{U}, \boldsymbol{P}$ such that

$$
\begin{aligned}
& \lim _{k \rightarrow \infty} \overline{\boldsymbol{e}}_{k}=\mathbf{0} \quad \text { for } \boldsymbol{v}_{k}=\mathbf{0} \\
& \left\|\overline{\boldsymbol{e}}_{k}\right\|_{l_{2}} \leq \mu\left\|\boldsymbol{v}_{k}\right\|_{l_{2}}, \quad \text { for } \boldsymbol{v}_{k} \neq \mathbf{0}, \overline{\boldsymbol{e}}_{0}=\mathbf{0} .
\end{aligned}
$$

In order to solve the above problem, it is sufficient to find a Lyapunov function $V_{k}$ such that (see, e.g., [48] for further details):

$$
\Delta V_{k}+\overline{\boldsymbol{e}}_{k}^{T} \overline{\boldsymbol{e}}_{k}-\mu^{2} \boldsymbol{v}_{k}^{T} \boldsymbol{v}_{k}<0, k=0, \ldots \infty
$$

where

$$
\begin{aligned}
\Delta V_{k} & =V_{k+1}-V_{k}, \\
V_{k} & =\overline{\boldsymbol{e}}_{k}^{T} \boldsymbol{P} \overline{\boldsymbol{e}}_{k}, \quad \boldsymbol{P} \succ \mathbf{0}, \\
\Delta V_{k} & =\overline{\boldsymbol{e}}_{k+1}^{T} \boldsymbol{P} \overline{\boldsymbol{e}}_{k+1}-\overline{\boldsymbol{e}}_{k}^{T} \boldsymbol{P} \overline{\boldsymbol{e}}_{k} .
\end{aligned}
$$

Consequently, using (36)

$$
\begin{aligned}
& \Delta V_{k}+\overline{\boldsymbol{e}}_{k}^{T} \overline{\boldsymbol{e}}_{k}-\mu^{2} \boldsymbol{v}_{k}^{T} \boldsymbol{v}_{k}= \\
& \overline{\boldsymbol{e}}_{k}^{T}\left(\boldsymbol{X}^{T} \boldsymbol{P} \boldsymbol{X}+\boldsymbol{I}-\boldsymbol{P}\right) \overline{\boldsymbol{e}}_{k}+ \\
& \overline{\boldsymbol{e}}_{k}^{T}\left(\boldsymbol{X}^{T} \boldsymbol{P} \boldsymbol{Y}\right) \boldsymbol{s}_{k}+ \\
& \overline{\boldsymbol{e}}_{k}^{T}\left(\boldsymbol{X}^{T} \boldsymbol{P} \boldsymbol{Z}\right) \boldsymbol{v}_{k}+ \\
& \boldsymbol{s}_{k}^{T}\left(\boldsymbol{Y}^{T} \boldsymbol{P} \boldsymbol{X}\right) \overline{\boldsymbol{e}}_{k}+ \\
& \boldsymbol{s}_{k}^{T}\left(\boldsymbol{Y}^{T} \boldsymbol{P} \boldsymbol{Y}\right) \boldsymbol{s}_{k}+ \\
& \boldsymbol{s}_{k}^{T}\left(\boldsymbol{Y}^{T} \boldsymbol{P} \boldsymbol{Z}\right) \boldsymbol{v}_{k}+ \\
& \boldsymbol{v}_{k}^{T}\left(\boldsymbol{Z}^{T} \boldsymbol{P} \boldsymbol{X}\right) \overline{\boldsymbol{e}}_{k}+ \\
& \boldsymbol{v}_{k}^{T}\left(\boldsymbol{Z}^{T} \boldsymbol{P} \boldsymbol{Y}\right) \boldsymbol{s}_{k}+ \\
& \boldsymbol{v}_{k}^{T}\left(\boldsymbol{Z}^{T} \boldsymbol{P} \boldsymbol{Z}-\mu^{2} \boldsymbol{I}\right) \boldsymbol{v}_{k}<\mathbf{0} .
\end{aligned}
$$

and by defining

$$
\overline{\boldsymbol{v}}_{k}=\left[\overline{\boldsymbol{e}}_{k}^{T}, \boldsymbol{s}_{k}^{T}, \boldsymbol{v}_{k}^{T}\right]^{T},
$$

it can be shown that (47) is equivalent to

$$
\overline{\boldsymbol{v}}_{k}^{T}\left[\begin{array}{ccc}
\boldsymbol{X}^{T} \boldsymbol{P} \boldsymbol{X}+\boldsymbol{I}-\boldsymbol{P} & \boldsymbol{X}^{T} \boldsymbol{P} \boldsymbol{Y} & \boldsymbol{X}^{T} \boldsymbol{P} \boldsymbol{Z} \\
\boldsymbol{Y}^{T} \boldsymbol{P} \boldsymbol{X} & \boldsymbol{Y}^{T} \boldsymbol{P} \boldsymbol{Y} & \boldsymbol{Y}^{T} \boldsymbol{P} \boldsymbol{Z} \\
\boldsymbol{Z}^{T} \boldsymbol{P} \boldsymbol{X} & \boldsymbol{Z}^{T} \boldsymbol{P} \boldsymbol{Y} & \boldsymbol{Z}^{T} \boldsymbol{P} \boldsymbol{Z}-\mu^{2} \boldsymbol{I}
\end{array}\right] \overline{\boldsymbol{v}}_{k} \prec \mathbf{0} .
$$

Following Assumption 1, it is evident that

$$
\boldsymbol{s}_{k}^{T} \boldsymbol{e}_{k} \leq \boldsymbol{e}_{k}^{T} \boldsymbol{M} \boldsymbol{e}_{k}=\frac{1}{2} \boldsymbol{e}_{k}^{T}\left(\boldsymbol{M}+\boldsymbol{M}^{T}\right) \boldsymbol{e}_{k} .
$$

By defining

$$
\boldsymbol{V}=\left[\begin{array}{ll}
\boldsymbol{I} & \mathbf{0}
\end{array}\right],
$$

the state estimation error is reflected by

$$
\boldsymbol{e}_{k}=\boldsymbol{V} \overline{\boldsymbol{e}}_{k}=\left[\begin{array}{ll}
\boldsymbol{I} & \mathbf{0}
\end{array}\right]\left[\begin{array}{c}
\boldsymbol{e}_{k} \\
\boldsymbol{e}_{f, k}
\end{array}\right]
$$


Using (52), inequality (50) can be written as

$$
\boldsymbol{s}_{k}^{T} \boldsymbol{V} \overline{\boldsymbol{e}}_{k} \leq \frac{1}{2} \overline{\boldsymbol{e}}_{k}^{T} \boldsymbol{V}^{T}\left(\boldsymbol{M}+\boldsymbol{M}^{T}\right) \boldsymbol{V} \overline{\boldsymbol{e}}_{k},
$$

which is equivalent to

$$
\frac{1}{2} \overline{\boldsymbol{e}}_{k}^{T} \boldsymbol{V}^{T}\left(\boldsymbol{M}+\boldsymbol{M}^{T}\right) \boldsymbol{V} \overline{\boldsymbol{e}}_{k}-\frac{1}{2} \boldsymbol{s}_{k}^{T} \boldsymbol{V} \overline{\boldsymbol{e}}_{k}-\frac{1}{2} \overline{\boldsymbol{e}}_{k}^{T} \boldsymbol{V}^{T} \boldsymbol{s}_{k} \geq \mathbf{0} .
$$

Thus, for any $\alpha>0$

$$
\alpha \overline{\boldsymbol{v}}_{k}^{T}\left[\begin{array}{ccc}
\boldsymbol{V}^{T}\left(\boldsymbol{M}+\boldsymbol{M}^{T}\right) \boldsymbol{V} & -\boldsymbol{V}^{T} & \mathbf{0} \\
-\boldsymbol{V} & \mathbf{0} & \mathbf{0} \\
\mathbf{0} & \mathbf{0} & \mathbf{0}
\end{array}\right] \overline{\boldsymbol{v}}_{k} \geq \mathbf{0} .
$$

Similarly, from Assumption 2 it can be shown that

$$
\boldsymbol{s}_{k}^{T} \boldsymbol{s}_{k} \leq \boldsymbol{e}_{k}^{T} \boldsymbol{M}^{T} \boldsymbol{M} \boldsymbol{e}_{k},
$$

which is equivalent to

$$
\overline{\boldsymbol{e}}_{k}^{T} \boldsymbol{V}^{T} \boldsymbol{M}^{T} \boldsymbol{M} \boldsymbol{V} \overline{\boldsymbol{e}}_{k}-\boldsymbol{s}_{k}^{T} \boldsymbol{s}_{k} \geq \mathbf{0}
$$

Thus, for any $\beta>0$

$$
\beta \overline{\boldsymbol{v}}_{k}^{T}\left[\begin{array}{ccc}
\boldsymbol{V}^{T} \boldsymbol{M}^{T} \boldsymbol{M} \boldsymbol{V} & \mathbf{0} & \mathbf{0} \\
\mathbf{0} & -\boldsymbol{I} & \mathbf{0} \\
\mathbf{0} & \mathbf{0} & \mathbf{0}
\end{array}\right] \overline{\boldsymbol{v}}_{k} \geq \mathbf{0} .
$$

Now, using the S-procedure for (49), (54) and (57) it can be concluded that

$$
\overline{\boldsymbol{v}}_{k}^{T}\left[\begin{array}{ccc}
\boldsymbol{X}^{T} \boldsymbol{P} \boldsymbol{X}+\boldsymbol{I}-\boldsymbol{P}+\alpha \boldsymbol{V}^{T}\left(\boldsymbol{M}+\boldsymbol{M}^{T}\right) \boldsymbol{V}+\beta \boldsymbol{V}^{T} \boldsymbol{M}^{T} \boldsymbol{M} \boldsymbol{V} & \boldsymbol{X}^{T} \boldsymbol{P} \boldsymbol{Y}-\alpha \boldsymbol{V}^{T} & \boldsymbol{X}^{T} \boldsymbol{P} \boldsymbol{Z} \\
\boldsymbol{Y}^{T} \boldsymbol{P} \boldsymbol{X}-\alpha \boldsymbol{V} & \boldsymbol{Y}^{T} \boldsymbol{P} \boldsymbol{Y}-\beta \boldsymbol{I} & \boldsymbol{Y}^{T} \boldsymbol{P} \boldsymbol{Z} \\
\boldsymbol{Z}^{T} \boldsymbol{P} \boldsymbol{X} & \boldsymbol{Z}^{T} \boldsymbol{P} \boldsymbol{Y} & \boldsymbol{Z}^{T} \boldsymbol{P} \boldsymbol{Z}-\mu^{2} \boldsymbol{I}
\end{array}\right] \overline{\boldsymbol{v}}_{k} \prec \mathbf{0} .
$$

Rewriting (58) as

$$
\left[\begin{array}{l}
\boldsymbol{X}^{T} \\
\boldsymbol{Y}^{T} \\
\boldsymbol{Z}^{T}
\end{array}\right] \boldsymbol{P}\left[\begin{array}{lll}
\boldsymbol{X} & \boldsymbol{Y} & \boldsymbol{Z}
\end{array}\right]+\left[\begin{array}{ccc}
\boldsymbol{I}-\boldsymbol{P}+\alpha \boldsymbol{V}^{T}\left(\boldsymbol{M}+\boldsymbol{M}^{T}\right) \boldsymbol{V}+\beta \boldsymbol{V}^{T} \boldsymbol{M}^{T} \boldsymbol{M} \boldsymbol{V} & -\alpha \boldsymbol{V}^{T} & \mathbf{0} \\
-\alpha \boldsymbol{V} & -\beta \boldsymbol{I} & \mathbf{0} \\
\mathbf{0} & \mathbf{0} & -\mu^{2} \boldsymbol{I}
\end{array}\right] \prec \mathbf{0}
$$

and applying Schur complements to (59) yields

$$
\left[\begin{array}{cccc}
\boldsymbol{I}-\boldsymbol{P}+\alpha \boldsymbol{V}^{T}\left(\boldsymbol{M}+\boldsymbol{M}^{T}\right) \boldsymbol{V}+\beta \boldsymbol{V}^{T} \boldsymbol{M}^{T} \boldsymbol{M} \boldsymbol{V} & -\alpha \boldsymbol{V}^{T} & \mathbf{0} & \boldsymbol{X}^{T} \boldsymbol{P} \\
-\alpha \boldsymbol{V} & -\beta \boldsymbol{I} & \mathbf{0} & \boldsymbol{Y}^{T} \boldsymbol{P} \\
\mathbf{0} & \mathbf{0} & -\mu^{2} \boldsymbol{I} & \boldsymbol{Z}^{T} \boldsymbol{P} \\
\boldsymbol{P} \boldsymbol{X} & \boldsymbol{P} \boldsymbol{Y} & \boldsymbol{P} \boldsymbol{Z} & -\boldsymbol{P}
\end{array}\right] \prec \mathbf{0} .
$$

Next, let us remind the following lemma [8]:

\section{Lemma 1}

The following statements are equivalent

1. There exists $\boldsymbol{X} \succ \mathbf{0}$ such that

$$
\boldsymbol{V}^{T} \boldsymbol{X} \boldsymbol{V}-\boldsymbol{W} \prec \mathbf{0},
$$

2. There exists $\boldsymbol{X} \succ \mathbf{0}$ such that

$$
\left[\begin{array}{cc}
-\boldsymbol{W} & \boldsymbol{V}^{T} \boldsymbol{U}^{T} \\
\boldsymbol{U} \boldsymbol{V} & \boldsymbol{X}-\boldsymbol{U}-\boldsymbol{U}^{T}
\end{array}\right] \prec \mathbf{0} .
$$


Applying Lemma 1 to (60) and then substituting

$$
\begin{gathered}
P X=P \bar{A}-P \bar{K} \bar{C}=P \bar{A}-N \bar{C}, \\
P Z=P \bar{W}-P \bar{K} \bar{V}=P \bar{W}-N \bar{V}
\end{gathered}
$$

where $\boldsymbol{N}=\boldsymbol{P} \overline{\boldsymbol{K}}$, lead to (40), which completes the proof.

Finally, the design procedure boils down to solving (40) for $M \in \mathbb{M}$ with respect to $\boldsymbol{N}, \boldsymbol{U}, \boldsymbol{P}, \alpha$, $\beta$ and then calculating

$$
\overline{\boldsymbol{K}}=\boldsymbol{P}^{-1} \boldsymbol{N}
$$

Note that $\mathbb{M}$, defined by (11), can be equivalently described by

$$
\mathbb{M}=\left\{\boldsymbol{M}(\alpha): \quad \boldsymbol{M}(\alpha)=\sum_{i=1}^{N} \alpha_{i} \boldsymbol{M}_{i}, \sum_{i=1}^{N} \alpha_{i}=1, \alpha_{i} \geq 0\right\}
$$

where $N=2^{n^{2}}$. Note that this is a general description, which does not take into account that some elements of $M$ may be constant. In such cases, $N$ is given by $N=2^{(n-c)^{2}}$, where $c$ stands for the number of constant elements of $M$.

Thus, solving (40) with respect to $\boldsymbol{N}, \boldsymbol{U}, \boldsymbol{P}, \alpha, \beta$ is equivalent to solving (for $i=1, \ldots, N$ )

$$
\left[\begin{array}{ccccc}
\boldsymbol{I}-\boldsymbol{P}+\alpha \boldsymbol{V}^{T}\left(\boldsymbol{M}_{i}+\boldsymbol{M}_{i}^{T}\right) \boldsymbol{V}^{T} & -\alpha \boldsymbol{V}^{T} & \mathbf{0} & \overline{\boldsymbol{A}}^{T} \boldsymbol{P}-\overline{\boldsymbol{C}}^{T} \boldsymbol{N}^{T} & \boldsymbol{V}^{T} \boldsymbol{M}_{i}^{T} \boldsymbol{U}^{T} \\
-\alpha \boldsymbol{V} & -\beta \boldsymbol{I} & \mathbf{0} & \boldsymbol{Y}^{T} \boldsymbol{P} & \mathbf{0} \\
\mathbf{0} & \mathbf{0} & -\mu^{2} \boldsymbol{I} & \overline{\boldsymbol{W}}^{T} \boldsymbol{P}-\overline{\boldsymbol{V}}^{T} \boldsymbol{N}^{T} & \mathbf{0} \\
\boldsymbol{P} \overline{\boldsymbol{A}}-\boldsymbol{N} \overline{\boldsymbol{C}} & \boldsymbol{P} \boldsymbol{P} & \boldsymbol{P} \overline{\boldsymbol{W}}-\boldsymbol{N} \boldsymbol{V} & -\boldsymbol{P}^{2} & \mathbf{0} \\
\boldsymbol{U} \boldsymbol{M}_{i} \boldsymbol{V} & \mathbf{0} & \mathbf{0} & \mathbf{0} & \beta \boldsymbol{I}-\boldsymbol{U}-\boldsymbol{U}^{T}
\end{array}\right] \prec \mathbf{0}
$$

and then determining

$$
\overline{\boldsymbol{K}}=\boldsymbol{P}^{-1} \boldsymbol{N} .
$$

Finally, it should be underlined that when (67) is feasible (for $i=1, \ldots, N$ ), then all its diagonal elements should be negative definite. In particular,

$$
\boldsymbol{I}-\boldsymbol{P}+\alpha \boldsymbol{V}^{T}\left(\boldsymbol{M}_{i}+\boldsymbol{M}_{i}^{T}\right) \boldsymbol{V}^{T} \prec \mathbf{0}
$$

Thus, when $\boldsymbol{V}^{T}\left(\boldsymbol{M}_{i}+\boldsymbol{M}_{i}^{T}\right) \boldsymbol{V}^{T} \preccurlyeq \mathbf{0}$, then it is evident that the feasibility of (67) can be obtained more easily than in the opposite case. However, when $\boldsymbol{V}^{T}\left(\boldsymbol{M}_{i}+\boldsymbol{M}_{i}^{T}\right) \boldsymbol{V}^{T} \succ \mathbf{0}$, then the optimization procedure will select $\alpha$ sufficiently close to zero in order to tackle this unappealing effect. A similar property underlines the one-sided Lipschitz condition-based design procedure proposed in [53]. However, as it was already mentioned, the proposed approach is perceived as a generalisation of the former one.

\section{ILLUSTRATIVE EXAMPLE}

Given a complete design procedure, the objective of this section is to provide a comprehensive performance study regarding the proposed approach along with a comparative study with respect to the Takagi-Sugeno (TS) based approach proposed by [4], shortly recalled in the Appendix. 


\subsection{Case study description}

Let us consider a twin rotor multiple-input multiple-output system (TRMS), portrayed in Fig. 1. This system is considered a challenging control problem because it includes high nonlinearities and couplings between axes, as can be seen in its model, described by the following differential equations

$$
\begin{aligned}
\frac{d \omega_{h}}{d t}= & \frac{k_{a} k_{1}}{J_{t r} R_{a}} u_{h}-\left(\frac{B_{t r}}{J_{t r}}+\frac{k_{a}^{2}}{J_{t r} R_{a}}\right) \omega_{h}-\frac{f_{1}\left(\omega_{h}\right)}{J_{t r}} \\
\frac{d \Omega_{h}}{d t}= & \frac{l_{t} f_{2}\left(\omega_{h}\right) \cos \theta_{v}-k_{o h} \Omega_{h}-f_{3}\left(\theta_{h}\right)+f_{6}\left(\theta_{v}\right)}{K_{D} \cos ^{2} \theta_{v}+K_{E} \sin ^{2} \theta_{v}+K_{F}} \\
& +\frac{k_{m} \omega_{v} \sin \theta_{v} \Omega_{v}\left(K_{D} \cos ^{2} \theta_{v}-K_{E} \sin ^{2} \theta_{v}-K_{F}-2 K_{E} \cos ^{2} \theta_{v}\right)}{\left(K_{D} \cos ^{2} \theta_{v}+K_{E} \sin ^{2} \theta_{v}+K_{F}\right)^{2}} \\
& +\frac{k_{m} \cos \theta_{v}\left(\frac{k_{a} k_{2}}{R_{a}} u_{v}-\left(B_{m r}+\frac{k_{a}^{2}}{R_{a}}\right) \omega_{v}-f_{4}\left(\omega_{v}\right)\right)}{J_{m r}\left(K_{D} \cos ^{2} \theta_{v}+K_{E} \sin ^{2} \theta_{v}+K_{F}\right)}, \\
\frac{d \theta_{h}}{d t}= & \Omega_{h}, \\
\frac{d \omega_{v}}{d t}= & \frac{k_{a} k_{2}}{J_{m r} R_{a}} u_{v}-\left(\frac{B_{m r}}{J_{m r}}+\frac{k_{a}^{2}}{J_{m r} R_{a}}\right) \omega_{v}-\frac{f_{2}\left(\omega_{v}\right)}{J_{m r}}, \\
\frac{d \Omega_{v}}{d t}= & \frac{l_{m} f_{5}\left(\omega_{v}\right)+k_{g} \Omega_{h} f_{5}\left(\omega_{v}\right) \cos \theta_{v}-k_{o v} \Omega_{v}}{J_{v}} \\
& +\frac{g\left(\left(K_{A}-K_{B}\right) \cos \theta_{v}-K_{C} \sin \theta_{v}\right)-\Omega_{h}^{2} K_{H} \sin \theta_{v} \cos \theta_{v}}{J_{v}} \\
& +\frac{k_{t}\left(\frac{k_{a} k_{1}}{R_{a}} u_{h}-\left(B_{t r}+\frac{k_{a}^{2}}{R_{a}}\right) \omega_{h}-f_{1}\left(\omega_{h}\right)\right)}{J_{v} J_{t r}} \\
\frac{d \theta_{v}}{d t}= & \Omega_{v},
\end{aligned}
$$

where $\omega_{h}$ is the rotational velocity of the tail rotor, $\Omega_{h}$ is the angular velocity of the TRMS around the vertical axis, $\theta_{h}$ is the yaw angle of the beam, $\omega_{v}$ is the rotational velocity of the main rotor, $\Omega_{v}$ is the angular velocity of the TRMS around the horizontal axis, and $\theta_{v}$ is the pitch angle of the beam. The system input vector is $\boldsymbol{u}=\left[u_{h}, u_{v}\right]^{T}$ while the system state vector is $\boldsymbol{x}=\left[\omega_{h}, \Omega_{h}, \theta_{h}, \omega_{v}, \Omega_{v}, \theta_{v}\right]^{T}$. The rest of the parameters are inherited from [38].

\subsection{Application of the proposed approach}

The non-linear model (70)-(75) has been discretized using Euler method with sampling time $T_{s}=0.01 \mathrm{~s}$, such that it can be expressed as (1)-(2) around an equilibrium point $\boldsymbol{x}_{e q}$, as follows

$$
\begin{aligned}
& \boldsymbol{x}_{k+1}=\boldsymbol{A}\left(\boldsymbol{x}_{e q}\right) \boldsymbol{x}_{k}+\boldsymbol{B}\left(\boldsymbol{x}_{e q}\right) \boldsymbol{u}_{k}+\boldsymbol{D} \boldsymbol{d}_{k}+\boldsymbol{g}\left(\boldsymbol{x}_{e q}, \boldsymbol{x}_{k}, \boldsymbol{u}_{k}\right)+\boldsymbol{B}\left(\boldsymbol{x}_{e q}\right) \boldsymbol{f}_{k}+\boldsymbol{W}_{1} \boldsymbol{w}_{k}, \\
& \boldsymbol{y}_{k}=\boldsymbol{C} \boldsymbol{x}_{k}+\boldsymbol{W}_{2} \boldsymbol{w}_{k},
\end{aligned}
$$

where $\boldsymbol{A}\left(\boldsymbol{x}_{e q}\right)$ and $\boldsymbol{B}\left(\boldsymbol{x}_{e q}\right)$ are the frozen system matrices at the equilibrium point, while the nonlinear function $\boldsymbol{g}\left(\boldsymbol{x}_{e q}, \boldsymbol{x}_{k}, \boldsymbol{u}_{k}\right)$ is defined as

$$
\boldsymbol{g}\left(\boldsymbol{x}_{e q}, \boldsymbol{x}_{k}, \boldsymbol{u}_{k}\right)=\left(\boldsymbol{A}\left(\boldsymbol{x}_{k}\right)-\boldsymbol{A}\left(\boldsymbol{x}_{e q}\right)\right) \boldsymbol{x}_{k},
$$




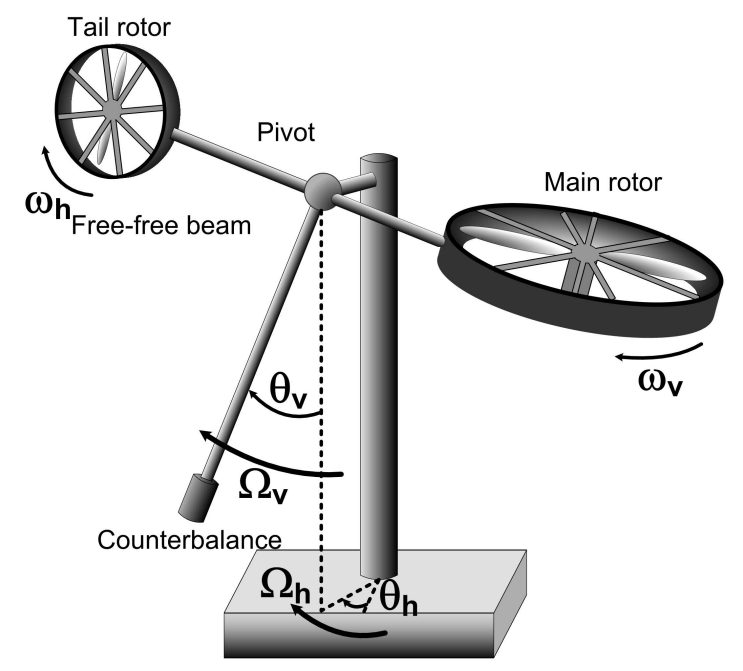

Figure 1. Components of the twin rotor MIMO system

In particular, for $\boldsymbol{x}_{e q}=0$, the matrices $\boldsymbol{A}\left(\boldsymbol{x}_{e q}\right)$ and $\boldsymbol{B}\left(\boldsymbol{x}_{e q}\right)$ are as follows:

$$
\boldsymbol{A}=\left[\begin{array}{cccccc}
0.8534 & 0 & 0 & 0 & 0 & 0 \\
0 & 0.9588 & -0.0352 & -0.0003 & 0 & 0.0014 \\
0 & 0.1000 & 1.0000 & 0 & 0 & 0 \\
0 & 0 & 0 & 0.9896 & 0 & 0 \\
0 & 0 & 0 & 0 & 0.8404 & -0.4116 \\
0 & 0 & 0 & 0 & 0.1000 & 1.0000
\end{array}\right], \boldsymbol{B}=\left[\begin{array}{cc}
0.2782 & 0 \\
0 & 0.0026 \\
0 & 0 \\
0 & 0.0845 \\
0 & 0 \\
0 & 0
\end{array}\right] .
$$

In a first step, it will be assumed that the whole state vector is directly measured, such that the remaining of the system matrices are as follows

$$
\begin{array}{cc}
\boldsymbol{C}=\boldsymbol{I}_{6} \\
& \boldsymbol{W}_{1}=0.01 \boldsymbol{I}_{6} \\
& \boldsymbol{W}_{2}=0.01 \boldsymbol{I}_{6}
\end{array}
$$

It is worth remarking that in this work the matrix $\boldsymbol{D}$ is used to show the relevant property of disturbance decoupling that characterizes the proposed strategy. In a real application to a TRMS, this matrix should be identified from available real data, in order to describe the possible disturbances affecting the system.

By varying each component of $\boldsymbol{x}_{k}$ within its possible domain, defined by the physical constraints of the TRMS, it is possible to obtain the matrices:

$$
\begin{gathered}
\boldsymbol{M}_{\max }=\left[\begin{array}{cccccc}
-0.0047 & 0 & 0 & 0 & 0 & 0 \\
0.1937 & 0.0682 & 0.0687 & -0.0002 & 0 & 0 \\
0 & 0 & 0 & 0 & 0 & 0 \\
0 & 0 & 0 & -0.0064 & 0 & 0 \\
0 & 0.0820 & 0 & 0.4531 & 0 & 0 \\
0 & 0 & 0 & 0 & 0 & 0
\end{array}\right], \\
\boldsymbol{M}_{\min }=\left[\begin{array}{cccccc}
-0.2705 & 0 & 0 & 0 & 0 & 0 \\
0.0032 & 0.0682 & 0.0583 & -0.0063 & 0 & 0 \\
0 & 0 & 0 & 0 & 0 & 0 \\
0 & 0 & 0 & -0.2050 & 0 & 0 \\
0 & -0.0820 & 0 & 0.0115 & 0 & 0 \\
0 & 0 & 0 & 0 & 0 & 0
\end{array}\right] .
\end{gathered}
$$


which contain all elements $\bar{a}_{i, j}$ and $\underline{a}_{i, j}, i, j=1, \ldots, n$ defining (11), respectively. Afterwards, taking into account all the possible combinations of elements in $\boldsymbol{M}_{\max }$ and $\boldsymbol{M}_{\min }, 128$ matrices $M_{i}$ are obtained (see (67)).

Using the design procedure described in Section 4, the following observer matrices are obtained with $\mu=10$ :

$$
\begin{aligned}
& \boldsymbol{N}=\left[\begin{array}{cccccc}
-1.4791 & -3.0394 & -1.0018 & -3.0841 & -0.5609 & -2.0607 \\
0.5946 & 2.9670 & 0.8084 & 3.0998 & 0.4621 & 1.6678 \\
-0.2378 & -1.4955 & -0.3327 & -1.3313 & -0.2603 & -0.9612 \\
-0.1301 & -1.0594 & -0.2551 & -1.5081 & -0.1532 & 0.5293 \\
-0.1279 & -0.7973 & -0.2482 & -0.7148 & -0.0020 & -0.5117 \\
-0.1756 & -1.3950 & -0.4622 & -1.0480 & -0.2533 & 0.8113
\end{array}\right] \\
& \boldsymbol{G}=\left[\begin{array}{cc}
0.2732 & -0.0077 \\
-0.0149 & -0.0205 \\
-0.0099 & -0.0154 \\
-0.0248 & 0.0461 \\
-0.0050 & -0.0077 \\
-0.0199 & -0.0307
\end{array}\right] \quad \boldsymbol{F}=\left[\begin{array}{cc}
5.7716 & 0.0994 \\
10.3356 & -1.9445 \\
3.3834 & -1.9873 \\
10.8163 & 10.1743 \\
1.9016 & -0.8907 \\
6.9611 & -3.9894
\end{array}\right]^{T} \\
& \boldsymbol{L}=\left[\begin{array}{cccccc}
1.6596 & 1.0117 & -0.3472 & -0.2922 & -0.1188 & -0.6338 \\
-0.0377 & -0.3653 & 0.2601 & -0.3522 & 0.0741 & 0.5515 \\
-0.0811 & 0.6204 & 0.6882 & -0.2874 & -0.0724 & -0.3208 \\
-0.1894 & 0.0546 & -0.4008 & 0.8391 & -0.2009 & -0.7650 \\
-0.0416 & 0.2796 & -0.0942 & -0.1449 & 0.6660 & -0.5812 \\
-0.1358 & 0.4240 & -0.1740 & -0.5575 & 0.0143 & 0.5530
\end{array}\right] \text {, } \\
& \boldsymbol{T}=\left[\begin{array}{cccccc}
0.9821 & -0.0536 & -0.0357 & -0.0893 & -0.0179 & -0.0714 \\
-0.0536 & 0.8393 & -0.1071 & -0.2679 & -0.0536 & -0.2143 \\
-0.0357 & -0.1071 & 0.9286 & -0.1786 & -0.0357 & -0.1429 \\
-0.0893 & -0.2679 & -0.1786 & 0.5536 & -0.0893 & -0.3571 \\
-0.0179 & -0.0536 & -0.0357 & -0.0893 & 0.9821 & -0.0714 \\
-0.0714 & -0.2143 & -0.1429 & 0.3571 & -0.0714 & 0.7143
\end{array}\right] \text {, } \\
& \boldsymbol{E}=\left[\begin{array}{llllll}
-0.0179 & -0.0536 & -0.0357 & -0.0893 & -0.0179 & -0.0714 \\
-0.0536 & -0.1607 & -0.1071 & -0.2679 & -0.0536 & -0.2143 \\
-0.0357 & -0.1071 & -0.0714 & -0.1786 & -0.0357 & -0.1429 \\
-0.0893 & -0.2679 & -0.1786 & -0.4464 & -0.0893 & -0.3571 \\
-0.0179 & -0.0536 & -0.0357 & -0.0893 & -0.0179 & -0.0714 \\
-0.0714 & -0.2143 & -0.1429 & -0.3571 & -0.0714 & -0.2857
\end{array}\right] \text {. }
\end{aligned}
$$

\subsection{Results}

In all the results presented in the following, the tail rotor and main rotor inputs $u_{h}(k)$ and $u_{v}(k)$ have been chosen as follows

$$
u_{h}(k)=0.5 \sin \left(\frac{k}{100}\right), \quad u_{v}(k)=0.2 \sin \left(\frac{k}{70}\right) .
$$

Moreover, a constant input disturbance $d_{k}=1$ has been considered, and $\boldsymbol{w}_{k}$ has been chosen as a uniformly distributed random vector, where each element takes values in the interval $[-0.1,0.1]$. Finally, the following initial state is considered for the system (70)-(75): $\boldsymbol{x}_{0}=[0,0.1,0,0,0,0.1]^{T}$, while the unknown input observer is initialized with $\boldsymbol{z}_{0}=\mathbf{0}$ and $\hat{\boldsymbol{f}}_{0}=\mathbf{0}$.

The observer is tested against selected actuator fault scenarios, i.e., additive faults in the tail motor $f_{h}(k)$ and additive faults in the main motor $f_{v}(k)$, described as follows: 


\section{Fault scenario S1:}

$$
\begin{aligned}
& f_{h}(k)=\left\{\begin{array}{cc}
-0.4 & 50 \leq k \leq 400 \\
0 & \text { otherwise }
\end{array}\right. \\
& f_{v}(k)=0
\end{aligned}
$$

Fault scenario S2:

$$
\begin{aligned}
& f_{h}(k)=0 \\
& f_{v}(k)=\left\{\begin{array}{cc}
-0.6 & 50 \leq k \leq 400 \\
0 & \text { otherwise }
\end{array}\right.
\end{aligned}
$$

Fault scenario S3:

$$
\begin{aligned}
& f_{h}(k)=\left\{\begin{array}{cc}
-0.5 & 50 \leq k \leq 400 \\
0 & \text { otherwise }
\end{array}\right. \\
& f_{v}(k)=\left\{\begin{array}{cc}
-0.5 & 50 \leq k \leq 400 \\
0 & \text { otherwise }
\end{array}\right.
\end{aligned}
$$

\section{Fault scenario S4:}

$$
\begin{aligned}
& f_{h}(k)=\left\{\begin{array}{cc}
-0.02(k-400) & 50 \leq k \leq 400, \\
0 & \text { otherwise }
\end{array}\right. \\
& f_{v}(k)=\left\{\begin{array}{cc}
0.01(k-400) & 50 \leq k \leq 400 \\
0 & \text { otherwise }
\end{array}\right.
\end{aligned}
$$

Figs. 2-5 present the results obtained with both the proposed approach (red dash-dot line) and the one based on a TS representation of the TRMS (blue dash line), recalled in the Appendix. Due to the disturbance $d_{k}$, the TS approach proposed by [4] fails in correctly estimating the state, as demonstrated by Fig. 2 and Fig. 3, where the state variables $\Omega_{v}$ and $\theta_{v}$, and their estimates, are depicted and compared. Despite [4] does not consider the problem of fault estimation, it could be demonstrated that (cf. [7] for further details)

$$
\hat{\boldsymbol{f}}_{k}=\left(\sum_{i=1}^{M} h_{i}\left(\boldsymbol{s}_{k-1}\right) \boldsymbol{B}^{i}\right)^{\dagger}\left[\hat{\boldsymbol{x}}_{k}-\sum_{i=1}^{M} h_{i}\left(\boldsymbol{s}_{k-1}\right)\left(\boldsymbol{A}^{i} \hat{\boldsymbol{x}}_{k-1}+\boldsymbol{B}^{i} \boldsymbol{u}_{k-1}\right)\right],
$$

where $(\cdot)^{\dagger}$ is a pseudoinverse of its argument, is a valid solution to this problem, that would work properly for $d_{k}=0$, but fails in presence of the disturbance $d_{k}$ because of the state estimation error, as shown in Fig. 4 and Fig. 5 for $\hat{\boldsymbol{f}}_{h}(k)$ and $\hat{\boldsymbol{f}}_{v}(k)$, respectively. On the other hand, the proposed unknown input observer (12)-(14) estimates the faults correctly.

Finally, to conclude the analysis of the proposed method, let us test its performance when some state variables are not directly measured. To this aim, let us consider the following output equation matrices:

$$
\boldsymbol{C}=\left[\begin{array}{llllll}
1 & 0 & 0 & 0 & 0 & 0 \\
0 & 1 & 0 & 0 & 0 & 0 \\
0 & 0 & 1 & 0 & 0 & 0 \\
0 & 0 & 0 & 1 & 0 & 0 \\
0 & 0 & 0 & 0 & 0 & 1
\end{array}\right], \quad \boldsymbol{W}_{2}=\left[\begin{array}{llllll}
1 & 0 & 0 & 0 & 0 & 0 \\
0 & 1 & 0 & 0 & 0 & 0 \\
0 & 0 & 1 & 0 & 0 & 0 \\
0 & 0 & 0 & 1 & 0 & 0 \\
0 & 0 & 0 & 0 & 1 & 0
\end{array}\right],
$$

such that the design procedure provides

$$
\boldsymbol{N}=\left[\begin{array}{cccccc}
-0.9611 & -0.0954 & -0.5940 & -0.1823 & -0.0073 & -0.0560 \\
0.0115 & -0.0637 & -0.4941 & -0.0128 & -0.0218 & -0.2201 \\
0.0095 & -0.0843 & -0.2652 & 0.0196 & -0.0145 & -0.1537 \\
-0.0522 & -0.0475 & -0.7950 & -1.1639 & -0.0364 & -0.1168 \\
-0.1151 & -0.1935 & -1.1699 & -0.6323 & 0.8331 & -1.1843 \\
0.2917 & 0.1709 & 1.6361 & 1.4959 & 0.0709 & 0.4034
\end{array}\right]
$$




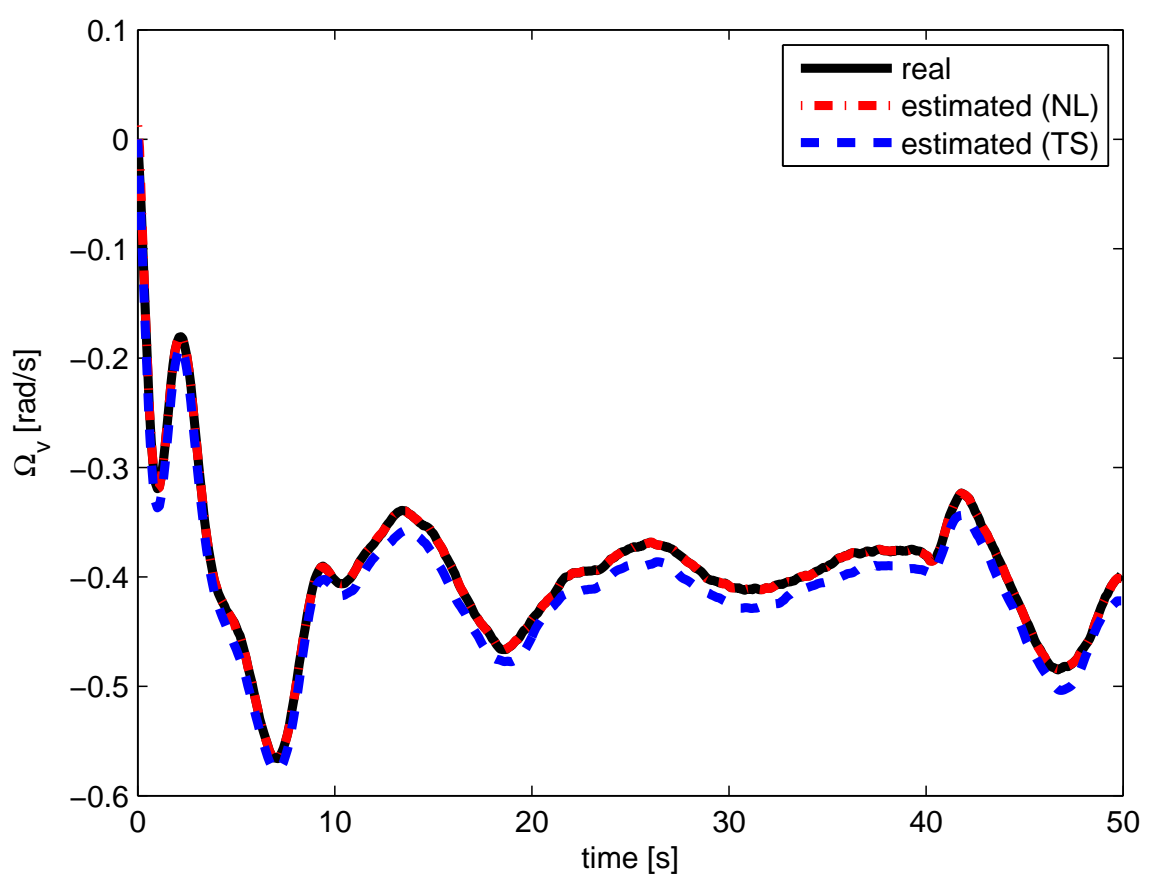

Figure 2. State variable $\Omega_{v}$ (black line) and its estimate (red/blue lines).

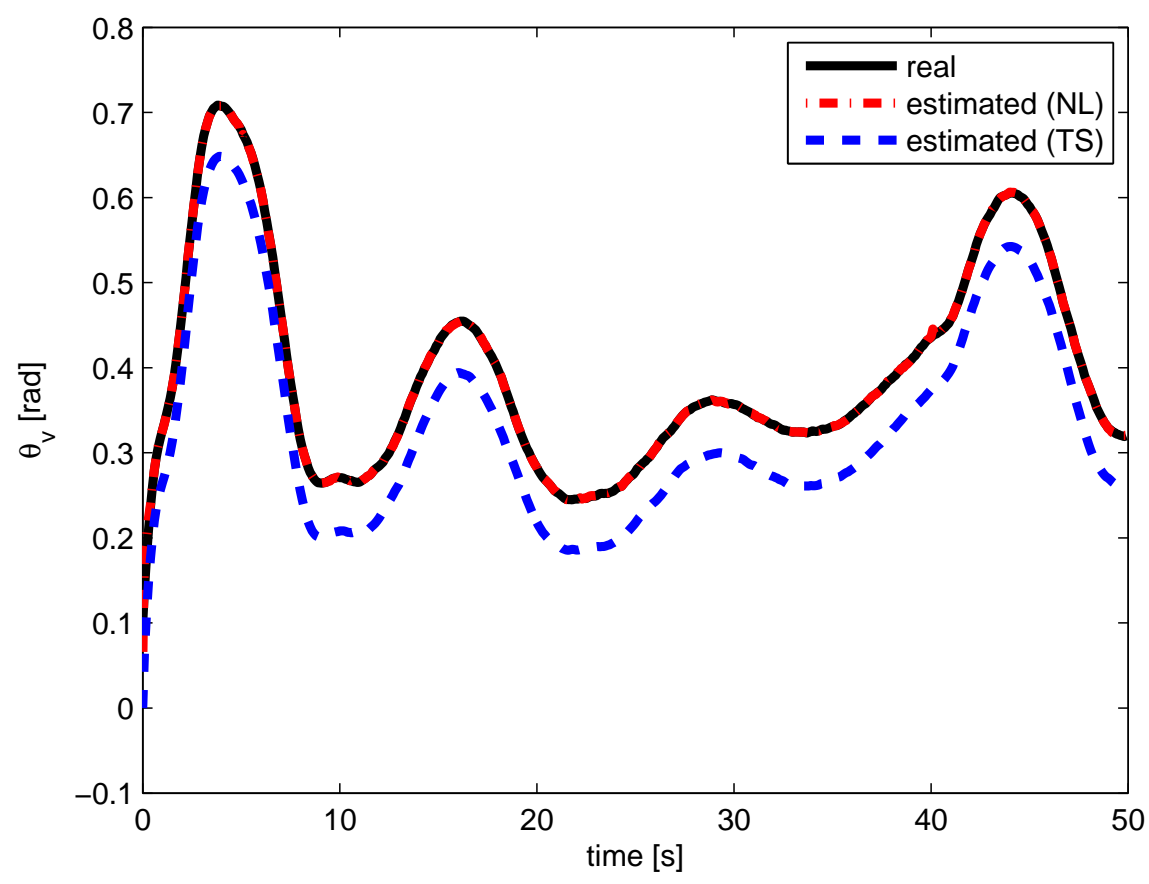

Figure 3. State variable $\theta_{v}$ (black line) and its estimate (red/blue lines).

$$
\boldsymbol{G}=\left[\begin{array}{cc}
0.2731 & -0.0078 \\
-0.0152 & -0.0209 \\
-0.0101 & -0.0156 \\
-0.0253 & 0.0454 \\
-0.0051 & -0.0078 \\
-0.0202 & -0.0313
\end{array}\right], \quad \boldsymbol{F}=\left[\begin{array}{cc}
3.6861 & 0.8946 \\
0.1892 & -1.9583 \\
1.5411 & -0.3188 \\
0.6863 & 15.0053 \\
-0.0727 & -3.1447
\end{array}\right]^{T}
$$




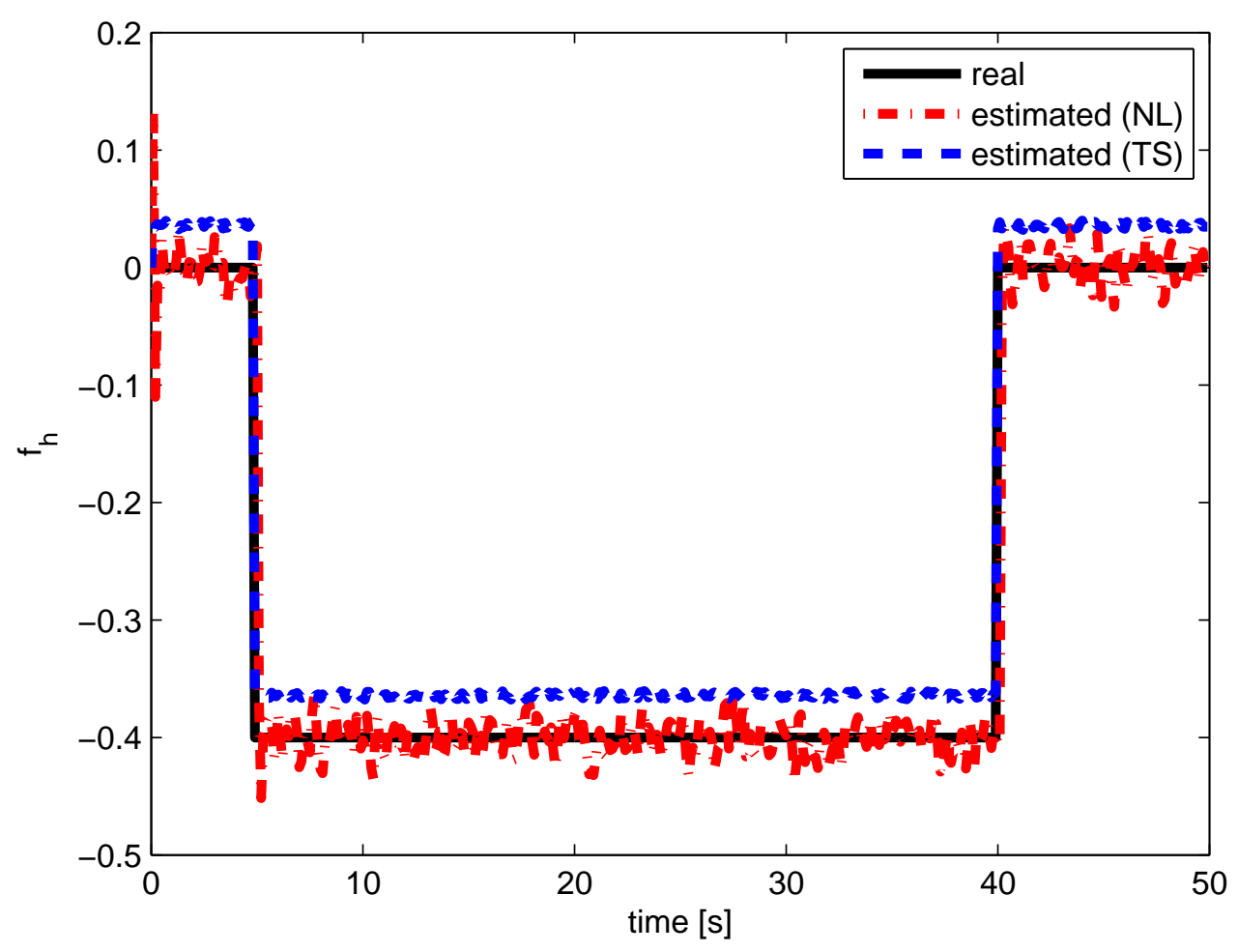

Figure 4. Fault in the tail motor $f_{h}$ (black line) and its estimate (red/blue lines).

$$
\begin{gathered}
\boldsymbol{L}=\left[\begin{array}{cccccc}
1.7340 & -0.1558 & 0.4295 & -0.2329 & -0.2770 \\
-0.0968 & 0.7383 & 0.2779 & -0.4513 & -0.1521 \\
-0.0643 & 0.0012 & 1.1489 & -0.3181 & -0.0868 \\
-0.1727 & -0.6744 & 0.3279 & 0.9667 & -0.8369 \\
-0.0840 & -0.4134 & 0.7682 & -0.3760 & -0.0347 \\
-0.1130 & 0.3276 & -1.2924 & -0.6520 & 1.2684
\end{array}\right] \\
\boldsymbol{T}=\left[\begin{array}{ccccccc}
0.9818 & -0.0545 & -0.0364 & -0.0909 & 0 & -0.0727 \\
-0.0545 & 0.8364 & -0.1091 & -0.2727 & 0 & -0.2182 \\
-0.0364 & -0.1091 & 0.9273 & -0.1818 & 0 & -0.1455 \\
-0.0909 & -0.2727 & -0.1818 & 0.5455 & 0 & -0.3636 \\
-0.0182 & -0.0545 & -0.0364 & -0.0909 & 1.0000 & -0.0727 \\
-0.0727 & -0.2182 & -0.1455 & -0.3636 & 0 & 0.7091
\end{array}\right] \\
\boldsymbol{E}=\left[\begin{array}{ccccccc}
-0.0182 & -0.0545 & -0.0364 & -0.0909 & -0.0727 \\
-0.0545 & -0.1636 & -0.1091 & -0.2727 & -0.2182 \\
-0.0364 & -0.1091 & -0.0727 & -0.1818 & -0.1455 \\
-0.0909 & -0.2727 & -0.1818 & -0.4545 & -0.3636 \\
-0.0182 & -0.0545 & -0.0364 & -0.0909 & -0.0727 \\
-0.0727 & -0.2182 & -0.1455 & -0.3636 & -0.2909
\end{array}\right]
\end{gathered}
$$

Fig. 6 presents the state variables and their estimates in the case of fault scenario S2. It can be seen that the state is correctly estimated. The same is true for the actuator faults, as shown in Fig. 7. Finally, for completeness of the presentation, the fault estimates in the cases of fault scenarios S3 and S4 are shown in Figs. 8 and 9, respectively, confirming the effectiveness of the proposed approach to cope with different fault behaviors. 


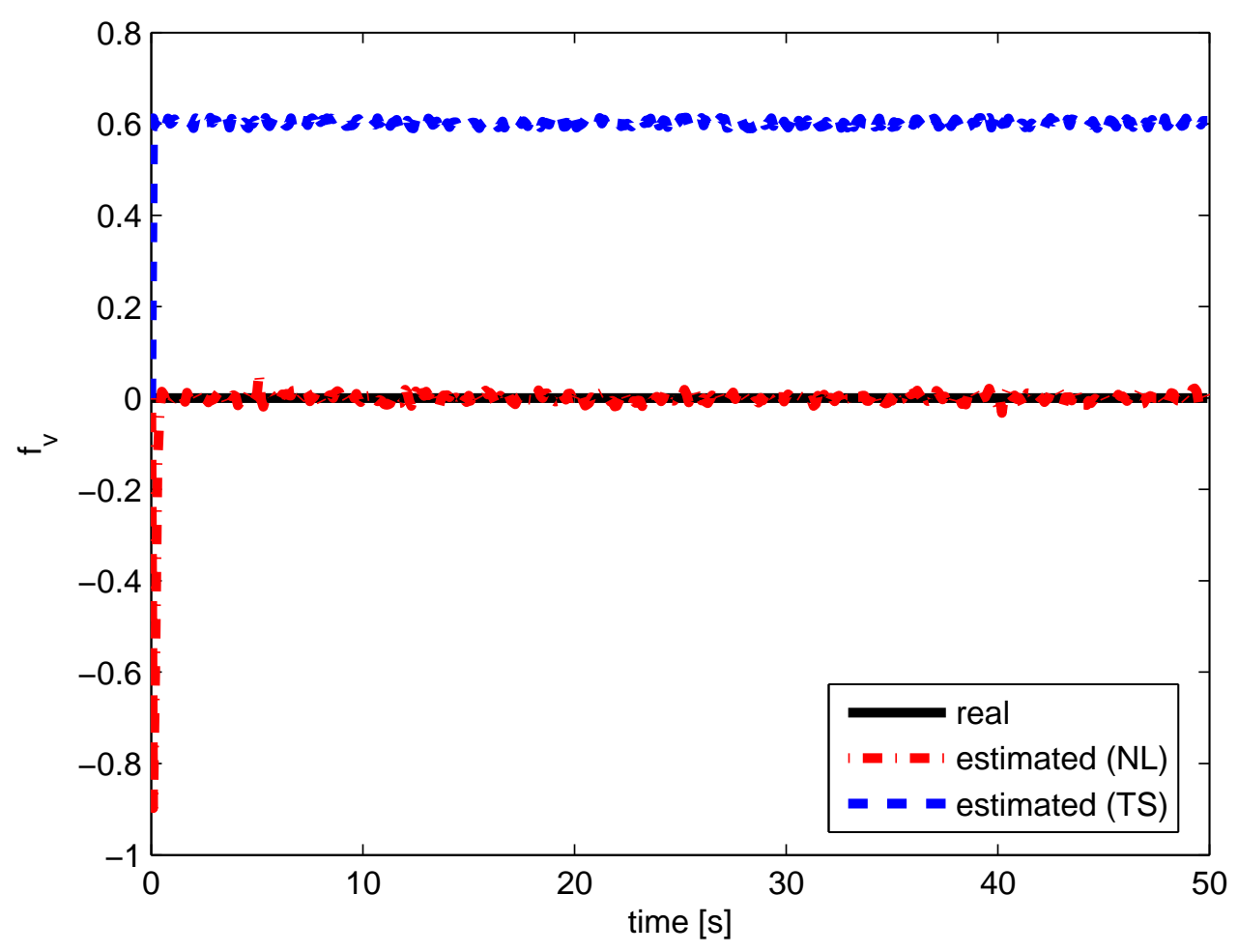

Figure 5. Fault in the main motor $f_{v}$ (black line) and its estimate (red/blue lines).

\title{
6. CONCLUSIONS
}

The main objective of this paper was to propose a novel approach which can be used for the estimation of an unknown fault signal for non-linear discrete-time stochastic systems. In particular, the fault and state estimation is achieved through the novel approach while robustness is attained with the $\mathcal{H}_{\infty}$ method and unknown input decoupling. Moreover, the description of non-linearity selected in this paper constitutes an interesting alternative to those presented in the literature. The proposed design procedure is relatively simple and boils down to solving a set of linear matrix inequalities. The presented results clearly exhibit the performance of the proposed approach when compared with competitive existing approaches in the application to a case study based on the TRMS. The main objective of further investigations is twofold: to design a robust adaptive threshold for the fault obtained with the proposed approach and to develop a robust FTC scheme which takes into account the fault estimates along with their uncertainties. Moreover, the proposed fault diagnosis and fault-tolerant approaches will be extended in such a way that the non-linear system will be described by the so-called state-space neural network. Taking into account the bounded nonlinearities of the neurons forming a neural network, the approach proposed in this paper seems to be well fitted to settle the above-mentioned task.

\begin{abstract}
APPENDIX
The approach proposed in this work is compared with the one recently developed in [4]. The authors of [4] have proposed observers for TS models subject to unknown inputs which influence states and outputs simultaneously. Since the problem considered in the present paper is to estimate the state and the actuator faults, the term in the output equation of [4], which may represent a sensor fault,
\end{abstract}



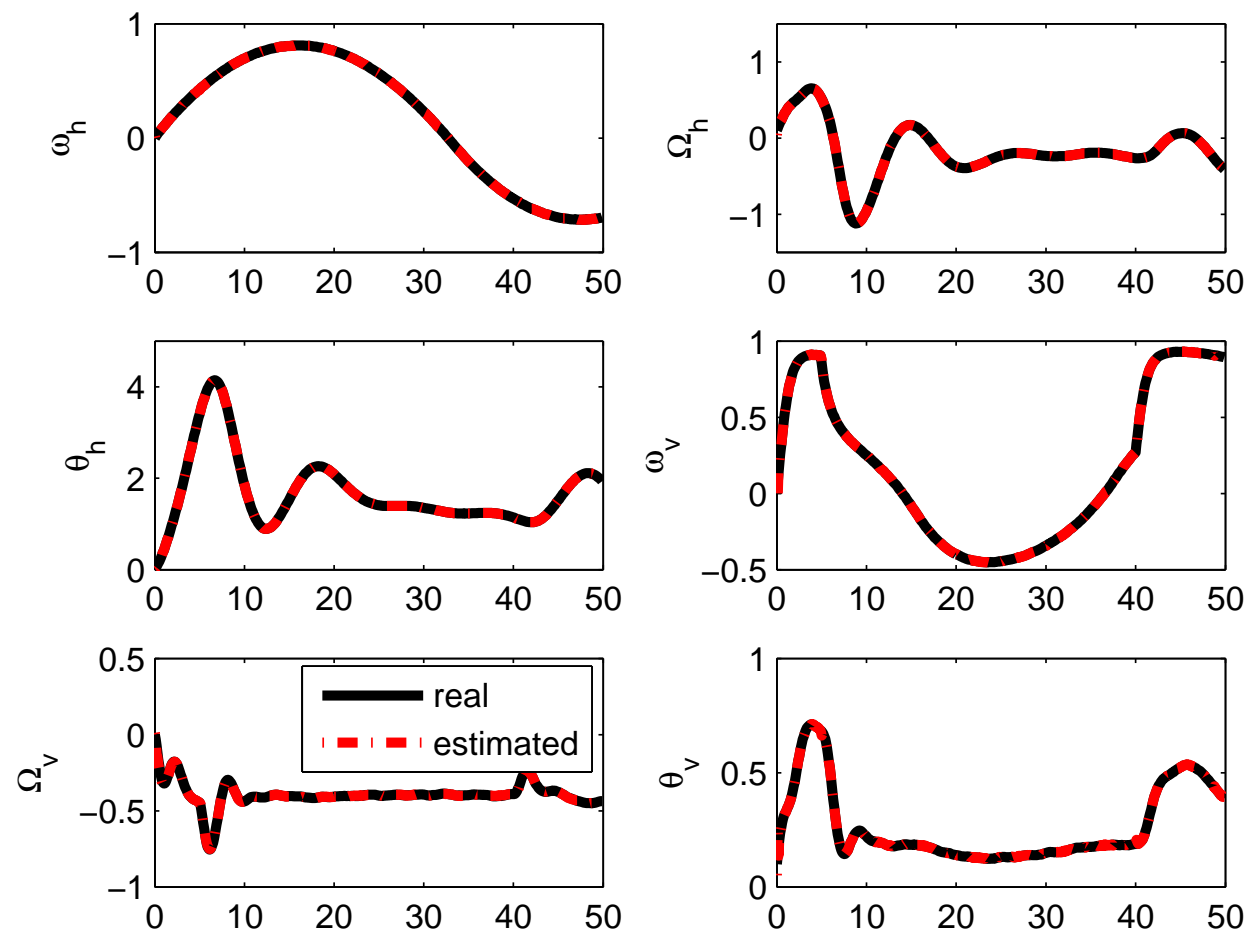

Figure 6. State variables (black lines) and their estimates using the proposed approach (red lines) in S2.
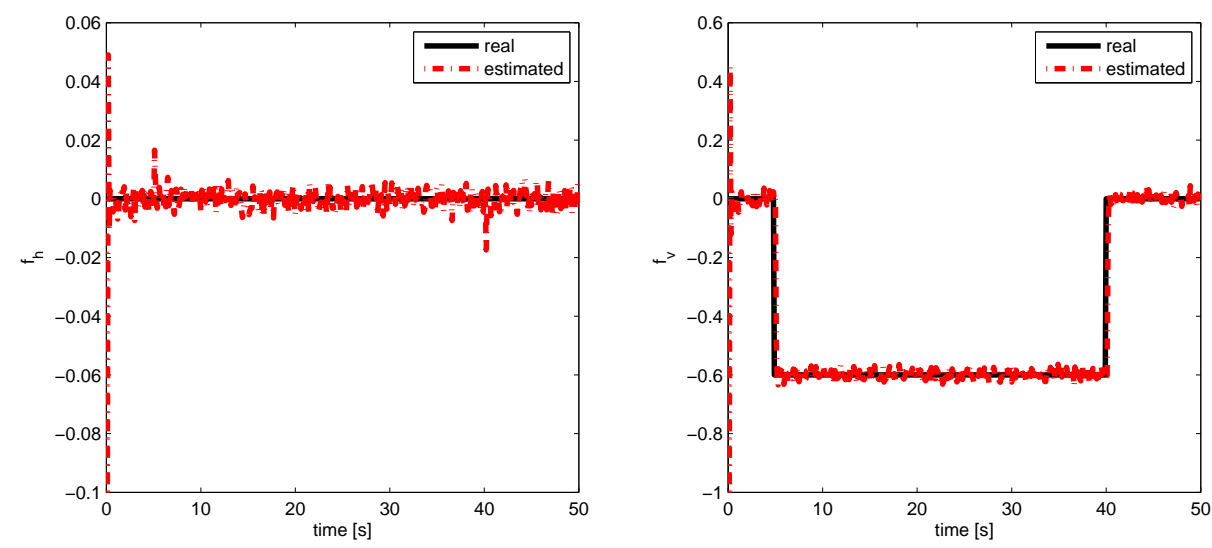

Figure 7. Faults (black lines) and their estimates using the proposed approach (red lines) in S2.

will be skipped in the following. Hence, the TS models considered in [4] are:

$$
\begin{aligned}
\boldsymbol{x}_{k+1} & =\sum_{i=1}^{M} h_{i}\left(\boldsymbol{s}_{k}\right)\left[\boldsymbol{A}^{i} \boldsymbol{x}_{k}+\boldsymbol{B}^{i} \boldsymbol{u}_{k}+\boldsymbol{B}^{i} \boldsymbol{f}_{k}+\boldsymbol{W}_{1}^{i} \boldsymbol{w}_{k}\right] \\
\boldsymbol{y}_{k} & =\boldsymbol{C} \boldsymbol{x}_{k}+\boldsymbol{W}_{2} \boldsymbol{w}_{k},
\end{aligned}
$$



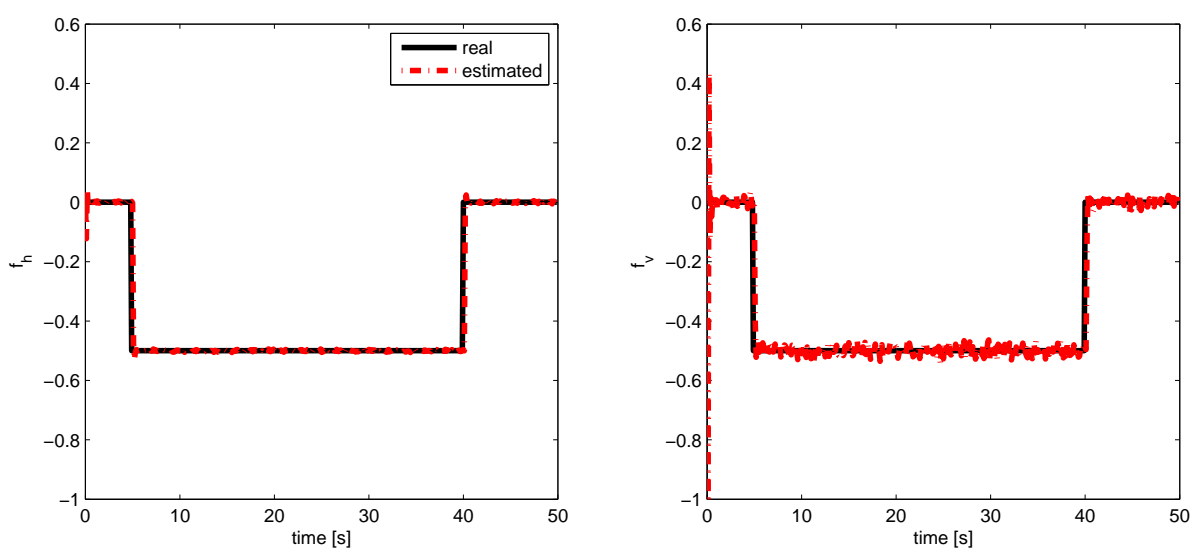

Figure 8. Faults (black lines) and their estimates using the proposed approach (red lines) in S3.
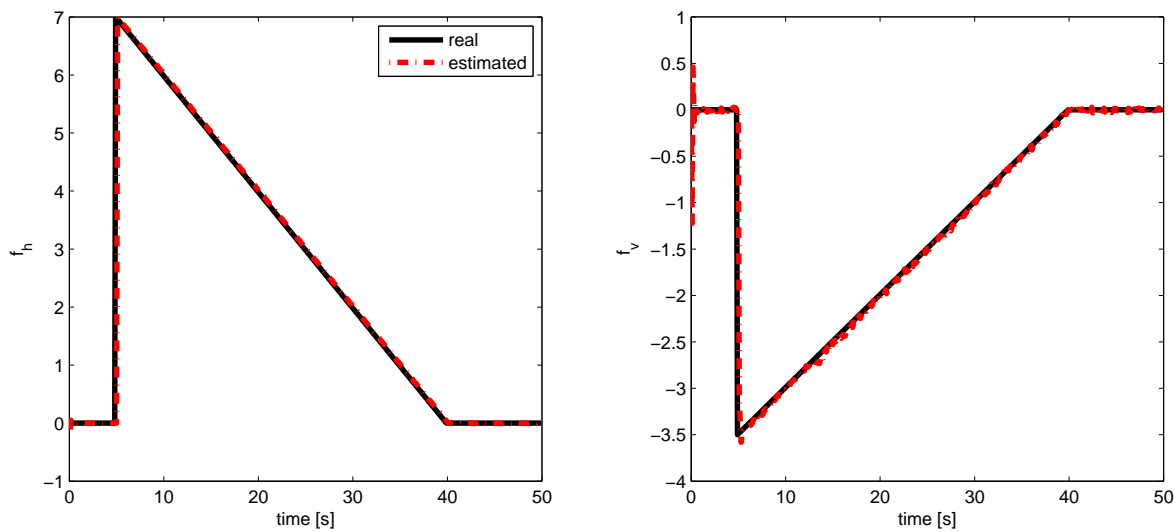

Figure 9. Faults (black lines) and their estimation using the proposed approach (red lines) in S4.

where $h_{i}\left(\boldsymbol{s}_{k}\right)$ are normalized rule firing strengths defined as

$$
h_{i}\left(\boldsymbol{s}_{k}\right)=\frac{\mathcal{T}_{j=1}^{p} \mu_{F_{j}^{i}}\left(s_{k}^{j}\right)}{\sum_{i=1}^{M}\left(\mathcal{T}_{j=1}^{p} \mu_{F_{j}^{i}}\left(s_{k}^{j}\right)\right)}
$$

and $\mathcal{T}$ denotes a $t$-norm (e.g., product). The term $\mu_{F_{j}^{i}}\left(s_{k}^{j}\right)$ is the grade of membership of the premise variable $s_{k}^{j}$. Moreover, the rule firing strengths $h_{i}\left(s_{k}\right)(i=1, \ldots, M)$ satisfy the following constraints

$$
\left\{\begin{array}{l}
\sum_{i=1}^{M} h_{i}\left(s_{k}\right)=1, \\
0 \leqslant h_{i}\left(s_{k}\right) \leqslant 1, \quad \forall i=1, \ldots, M .
\end{array}\right.
$$

while the associated unknown input observer is

$$
\begin{aligned}
\boldsymbol{z}_{k+1} & =\sum_{i=1}^{M} h_{i}\left(\boldsymbol{s}_{k}\right)\left[\boldsymbol{N}^{i} \boldsymbol{z}_{k}+\boldsymbol{G}^{i} \boldsymbol{u}_{k}+\boldsymbol{L}^{i} \boldsymbol{y}_{k}\right], \\
\hat{\boldsymbol{x}}_{k} & =\boldsymbol{z}_{k}+\boldsymbol{E} \boldsymbol{y}_{k} .
\end{aligned}
$$

The overall design procedure, which guarantees that the state estimation error $\boldsymbol{e}_{k}$ converges asymptotically to zero, can be reduced to solving a relatively simple set of LMIs. 
For the sake of comparison, let us notice that the TS models (79) do not contain the disturbance term $\boldsymbol{d}_{k}$, such that [4] does not achieve a decoupling with respect to this term, contrarily to the approach proposed in the present paper.

\section{ACKNOWLEDGMENTS}

The authors would like to express their sincere gratitude to the referees, whose constructive comments contributed significantly towards the current shape of the paper.

\section{REFERENCES}

1. M. Abbaszadeh and H.J. Marquez. LMI optimization approach to robust $\mathcal{H}_{\infty}$ observer design and static output feedback stabilization for non-linear uncertain systems. International Journal of Robust and Nonlinear Control, 19(3):313-340, 2008.

2. F. Amato, C. Cosentino, M. Mattei, and G. Paviglianiti. A mixed direct/functional redundancy scheme for the fdi on a small commercial aircraft. In Proceedings of the SAFEPROCESSO3, IFAC symposium, pages 331-339, 2003.

3. M. Blanke, M. Kinnaert, J. Lunze, and M. Staroswiecki. Diagnosis and Fault-Tolerant Control. Second Edition. Springer-Verlag Berlin Heidelberg, 2006.

4. M. Chadli and H.R. Karimi. Robust observer design for unknown inputs Takagi-Sugeno models. IEEE Transactions on Fuzzy Systems, 21(1):158-164, 2013.

5. J. Chen and R.J. Patton. Robust Model-Based Fault Diagnosis for Dynamic Systems. Kluwer Academic Publishers, 1999.

6. D. Ichalal and B. Marx and J. Ragot and D. Maquin. Fault detection, isolation and estimation for Takagi-Sugeno nonlinear systems. Journal of The Franklin Institute, 351(7):36513676, 2014.

7. S. de Oca, V. Puig, M. Witczak, and L. Dziekan. Fault-tolerant control strategy for actuator faults using LPV techniques: Application to a two degree of freedom helicopter. International Journal of Applied Mathematics and Computer Science, 22(1):161-171, 2012.

8. M.C. de Oliveira, J. Bernussou, and J.C. Geromel. A new discrete-time robust stability condition. Systems and Control Letters, 37(4):261-265, 1999.

9. S.X. Ding. Model-based Fault Diagnosis Techniques: Design Schemes, Algorithms, and Tools. Springer-Verlag, Berlin, Heidelberg, 2008.

10. L. Dziekan, M. Witczak, and J. Korbicz. Active fault-tolerant control design for Takagi-Sugeno fuzzy systems. Bulletin of the Polish Academy of Sciences: Technical Sciences, 59(1):93-102, 2011.

11. P. M. Frank and T. Marcu. Diagnosis strategies and systems. principles, fuzzy and neural approaches. In Intelligent Systems and Interfaces (Teodorescu H. N., Mlynek D., Kandel A. and Zimmermann H. J. (Eds.). Kluwer Academic Publishers, Boston, 2000.

12. J. Gertler. Fault Detection and Diagnosis in Engineering Systems. Marcel Dekker, New York, 1998.

13. S. Gillijns and B. De Moor. Unbiased minimum-variance input and state estimation for linear discrete-time systems. Automatica, 43:111-116, 2007.

14. M. Kinnaert H. Hammouri and E. H. El Yaagoubi. Observer-based approach to fault detection and isolation for nonlinear systems. IEEE Trans. on Automatic Control, 44:1879-1884, 1999.

15. H. Hammouri, P. Kabore, and M. Kinnaert. A geometric approach to fault detection and isolation for bilinear systems. IEEE Transactions on Automatic Control, 46(9):1451-1455, 2001.

16. H. Hammouri, P. Kabore, S. Othman, and J. Biston. Observer-based approach to fault detection and isolation for nonlinear systems. IEEE Transactions on Automatic Control, 44(10):1879-1884, 1999.

17. H. Hammouri, P. Kabore, S. Othman, and J. Biston. Failure diagnosis and nonlinear observer. application to a hydraulic process. Journal of The Franklin Institute, 339(4-5):455-478, 2002.

18. R. Isermann. Fault Diagnosis Systems. An Introduction from Fault Detection to Fault Tolerance. Springer-Verlag, New York, 2006.

19. R. Isermann. Fault Diagnosis Applications: Model Based Condition Monitoring, Actuators, Drives, Machinery, Plants, Sensors, and Fault-tolerant Systems. Springer-Verlag, Berlin, 2011.

20. R. Kabore and H. Wang. Design of fault diagnosis filters and fault tolerant control for a class of nonlinear systems. IEEE Transactions on Automatic Control, 46(11):1805-1809, 2001.

21. J. Y. Keller and M. Darouach. Two-stage Kalman estimator with unknown exogenous inputs. Automatica, 35(2):339-342, 1999.

22. K. Kemir, F. Ben Hmida, J. Ragot, and M. Gossa. Novel optimal recursive filter for state and fault estimation of linear systems with unknown disturbances. International Journal of Applied Mathematics and Computer Science, 21(4):629-638, 2011.

23. J. Korbicz, J. Kościelny, Z. Kowalczuk, and W. Cholewa (Eds.). Fault Diagnosis. Models, Artificial Intelligence, Applications. Springer-Verlag, Berlin, 2004.

24. H. Li and M. Fu. A linear matrix inequality approach to robust $\mathcal{H}_{\infty}$ filtering. IEEE Transactions on Signal Processing, 45(9):2338-2350, 1997.

25. Mattei M., Paviglianiti G., and Scordamaglia V. Nonlinear observers with $\mathcal{H}_{\infty}$ performance for sensor fault detection and isolation: a linear matrix inequality design procedure. Control Engineering Practice, (13):12711281, 2006. 
26. M. Mahmoud, J. Jiang, and Y. Zhang. Active Fault Tolerant Control Systems: Stochastic Analysis and Synthesis. Springer-Verlag, Berlin, 2003.

27. H.J. Marquez. Nonlinear control systems. Analysis and Design. Wiley, New Jersey, 2003.

28. M. Mrugalski. An unscented Kalman filter in designing dynamic GMDH neural networks for robust fault detection. International Journal of Applied Mathematics and Computer Science, 23(1):157-169, 2013.

29. E.G. Nobrega, M.O. Abdalla, and K.M. Grigoriadis. Robust fault estimation of unceratain systems using an LMIbased approach. International Journal of Robust and Nonlinear Control, 18(7):1657-1680, 2008.

30. H. Noura, D. Theilliol, J. Ponsart, and A. Chamseddine. Fault-tolerant Control Systems: Design and Practical Applications. Springer-Verlag, Berlin, 2003.

31. R.J. Patton, L. Chen, and S. Klinkhieo. Lpv approach to friction estimation as a fault diagnosis problem. In 15th International Conference on Methods and Models in Automation and Robotics (MMAR), 2010, Miedzyzdroje, Poland, pages 296-301. IEEE, 2010.

32. R.J. Patton and S. Klinkhieo. Actuator fault estimation and compensation based on an augmented state observer approach. In Proceedings of the 48th IEEE Conference on Decision and Control, 2009 held jointly with the 2009 28th Chinese Control Conference. CDC/CCC 2009, Shanghai, China, pages 8482-8487. IEEE, 2009.

33. C. De Persis and A. Isidori. A geometric approach to nonlinear fault detection and isolation. Automatic Control, IEEE Transactions on, 46(6):853-865, 2001.

34. C. De Persis, R. De Santis, and A. Isidori. An $\mathcal{H}_{\infty}$-suboptimal fault detection filter for bilinear systems. In Proceedings of the Nonlinear control in the Year 2000, pages 331-339, 2000.

35. C. De Persis, R. De Santis, and A. Isidori. Nonlinear actuator fault detection and isolation for a vtol aircraft. In Proceedings of the 2001 American Control Conference, June 25-27, 2001.

36. A. M. Pertew, H. J. Marquez, and Q. Zhao. $\mathcal{H}_{\infty}$ synthesis of unknown input observers for non-linear Lipschitz systems. International Journal of Control, 78(15):1155-1165, 2005.

37. R. Rajamani. Observers for Lipschitz non-linear systems. IEEE Transactions on Automatic Control, 43(3):397401, 1998.

38. D. Rotondo, F. Fatiha Nejjari, and V. Puig. Quasi-lpv modeling, identification and control of atwin rotor mimo system. Control Engineering Practice, 21(6):829846, 2013.

39. D. Rotondo, F. Nejjari, and V. Puig. A virtual actuator and sensor approach for fault tolerant control of lpv systems. Journal of Process Control, 24(3):203-222, 2014.

40. M. Sami and R.J. Patton. A multiple-model approach to fault tolerant tracking control for non-linear systems. In 20th Mediterranean Conference on Control \& Automation (MED), 2012, Barcelona, Spain, pages 498-503. IEEE, 2012.

41. Mara M. Seron and Jos A. De Don. Robust fault estimation and compensation for $\{$ LPV $\}$ systems under actuator and sensor faults. Automatica, 52(0):294 - 301, 2015.

42. D.M. Stipanovic and D.D. Siljak. Robust stability and stabilization of discrete-time non-linear: the LMI approach. International Journal of Control, 74(5):873-879, 2001.

43. X. Sun and R.J. Patton. Robust actuator multiplicative fault estimation with unknown input decoupling for a wind turbine system. In Conference on Control and Fault-Tolerant Systems (SysTol), 2013, Nice, France, pages 263-268. IEEE, 2013.

44. X. Sun, Ron J Patton, and Philippe Goupil. Robust adaptive fault estimation for a commercial aircraft oscillatory fault scenario. In UKACC International Conference on Control (CONTROL), 2012, Cardif, United Kingdom, pages 595-600. IEEE, 2012.

45. Seyed Mojtaba Tabatabaeipour and Thomas Bak. Robust observer-based fault estimation and accommodation of discrete-time piecewise linear systems. Journal of the Franklin Institute, 351(1):277 - 295, 2014.

46. K.C. Veluvolu, M.Y Kim, and D. Lee. Nonlinear sliding mode high-gain observers for fault estimation. International Journal of Systems Science, 42(7):1065-1074, 2011.

47. M. Witczak. Modelling and Estimation Strategies for Fault Diagnosis of Non-linear Systems. Springer-Verlag, Berlin, 2007.

48. M. Witczak. Fault Diagnosis and Fault-Tolerant Control Strategies for Non-Linear Systems: Analytical and Soft Computing approaches. Springer International Publishing, Heidelberg, Germany, 2014.

49. M. Witczak, V. Puig, and S. de Oca. A fault-tolerant control strategy for non-linear discrete-time systems: application to the twin-rotor system. International Journal of Control, 86:in print, 2013.

50. A. Zemouche and M. Boutayeb. Observer design for Lipschitz non-linear systems: the discrete time case. IEEE Transactions on Circuits and Systems - II:Express Briefs, 53(8):777-781, 2006.

51. A. Zemouche, M. Boutayeb, and G. Iulia Bara. Observer for a class of Lipschitz systems with extension to $\mathcal{H}_{\infty}$ performance analysis. Systems and Control Letters, 57(1):18-27, 2008.

52. X. Zhang, M.M. Polycarpou, and T. Prisini. Fault diagnosis of a class of nonlinear uncertain systems with Lipschitz nonlinearities using adaptive estimation. Automatica, 46(2):290-299, 2010

53. Y. Zhao, J. Tao, and N. Shi. A note on observer design for one-sided lipschitz nonlinear systems. Systems and Control Letters, 59(1):66-71, 2010. 